\title{
The conformational state of hERG1 channels determines integrin association, downstream signaling, and cancer progression
}

2017 (c) The Authors, some rights reserved; exclusive licensee American Association for the Advancement of Science.

\author{
Andrea Becchetti, ${ }^{1}$ Silvia Crescioli, ${ }^{2}$ Francesca Zanieri, ${ }^{2}$ Giulia Petroni, ${ }^{2}$ Raffaella Mercatelli, ${ }^{3}$ \\ Stefano Coppola, ${ }^{4}$ Luca Gasparoli, ${ }^{2}$ Massimo D'Amico, ${ }^{5}$ Serena Pillozzi, ${ }^{2}$ Olivia Crociani, ${ }^{2}$ \\ Matteo Stefanini, ${ }^{5}$ Antonella Fiore, ${ }^{2}$ Laura Carraresi, ${ }^{5}$ Virginia Morello, ${ }^{6 *}$ Sagar Manoli, ${ }^{2}$ \\ Maria Felice Brizzi, ${ }^{7}$ Davide Ricci, ${ }^{8}$ Mauro Rinaldi, ${ }^{8}$ Alessio Masi, ${ }^{2 \dagger}$ Thomas Schmidt, $^{4}$ \\ Franco Quercioli, ${ }^{3}$ Paola Defilippi, ${ }^{4}$ Annarosa Arcangeli ${ }^{2 \ddagger}$
}

Ion channels regulate cell proliferation, differentiation, and migration in normal and neoplastic cells through cell-cell and cell-extracellular matrix (ECM) transmembrane receptors called integrins. $\mathrm{K}^{+}$flux through the human ether-à-gogo-related gene 1 (hERG1) channel shapes action potential firing in excitable cells such as cardiomyocytes. Its abundance is often aberrantly high in tumors, where it modulates integrin-mediated signaling. We found that hERG1 interacted with the $\boldsymbol{\beta}_{1}$ integrin subunit at the plasma membrane of human cancer cells. This interaction was not detected in cardiomyocytes because of the presence of the hERG1 auxiliary subunit KCNE1 (potassium voltage-gated channel subfamily E regulatory subunit 1 ), which blocked the $\boldsymbol{\beta}_{1}$ integrin-hERG1 interaction. Although open hERG1 channels did not interact as strongly with $\beta_{1}$ integrins as did closed channels, current flow through hERG1 channels was necessary to activate the integrin-dependent phosphorylation of $\mathrm{Tyr}^{397}$ in focal adhesion kinase (FAK) in both normal and cancer cells. In immunodeficient mice, proliferation was inhibited in breast cancer cells expressing forms of hERG1 with impaired $\mathrm{K}^{+}$flow, whereas metastasis of breast cancer cells was reduced when the $\mathrm{hERG} 1 / \boldsymbol{\beta}_{1}$ integrin interaction was disrupted. We conclude that the interaction of $\boldsymbol{\beta}_{1}$ integrins with hERG1 channels in cancer cells stimulated distinct signaling pathways that depended on the conformational state of hERG1 and affected different aspects of tumor progression.

\section{INTRODUCTION}

Cell adhesion to the extracellular matrix (ECM) is critical to various processes, including cell migration and synaptic plasticity, and the decision between proliferation and differentiation $(1,2)$. Both voltage- and ligand-gated ion channels regulate how cells respond to the ECM by interacting with integrin receptors $(3,4)$. Integrin receptors are transmembrane proteins consisting of various $\alpha$ and $\beta$ subunits. In mammals, they can form more than 20 different heterodimers with specific ECM binding patterns $(5,6)$. Under resting conditions, integrin receptors reside in a folded low-affinity conformational state. Upon activation by extracellular or intracellular ligands or binding partners, they shift to a highaffinity extended conformation $(7,8)$, which mediates both "outside-in" and "inside-out" signaling (5). An important early signal triggered by integrin engagement is the phosphorylation of focal adhesion kinase (FAK), which, in turn, regulates downstream signaling (9).

\footnotetext{
${ }^{1}$ Department of Biotechnology and Biosciences, University of Milano-Bicocca, Piazza della Scienza 2, 20126 Milano, Italy. ${ }^{2}$ Department of Experimental and Clinical Medicine, University of Firenze, Viale G.B. Morgagni 50, 50134 Firenze, Italy. ${ }^{3}$ Consiglio Nazionale delle Ricerche-Istituto Nazionale di Ottica, Via N. Carrara 1, 50019 Sesto Fiorentino, Italy. ${ }^{4}$ Physics of Life Processes, Huygens-Kamerlingh Onnes Laboratory, Leiden University, Niels Bohrweg 2, 2333 CA Leiden, Netherlands. ${ }^{5}$ Di.V.A.L. Toscana SRL, Via Madonna del Piano 6, 50019 Sesto Fiorentino, Italy. ${ }^{6}$ Department of Molecular Biotechnology and Health Sciences, University of Torino, Via Nizza 52, 10126 Torino, Italy. ${ }^{7}$ Department of Medical Sciences, University of Torino, Corso Dogliotti 14, 10126 Torino, Italy. ${ }^{8}$ Department of Surgical Sciences, University of Torino, Corso Dogliotti 14, 10126 Torino, Italy.

*Present address: Institute for Cancer Research and Treatment, Strada Provinciale 142, 10060 Candiolo, Italy.

†Present address: Section of Pharmacology and Toxicology, Department of Neuroscience, Psychology, Drug Research and Child Health, University of Firenze, Viale Pieraccini 6, 50134 Firenze, Italy.

‡Corresponding author. Email: annarosa.arcangeli@unifi.it
}

A common molecular partner of integrin receptors is the human ether-à-go-go-related gene 1 protein (hERG1, also known as Kv11.1). In cardiomyocytes, hERG1 mediates the cardiac repolarizing current $I_{\mathrm{kr}}$ (10) in association with accessory subunits such as the potassium voltagegated channel subfamily E regulatory subunit 1 (KCNE1, also known as MinK1). Moreover, hERG1 regulates excitability in the central nervous system, endocrine cells, and smooth muscle $(11,12)$. However, information about the interaction between integrin receptors and hERG1 is available only for neoplastic cells. The abundance of hERG1 is often aberrantly high in human cancers, and this channel is implicated in different stages of neoplastic progression, such as cell proliferation and survival, invasiveness, and neoangiogenesis (12-14). Because these processes are regulated by cell adhesion to the ECM, the cross-talk between integrin receptors and hERG1 may constitute a unifying modulatory mechanism of the cellular response to the microenvironment in both normal and tumor tissue $(4,15)$.

Integrin receptors and ion channels communicate by diffusible signals (16-20) as well as by forming macromolecular complexes (21-25). In particular, the $\beta_{1}$ integrin-mediated adhesion to fibronectin activates hERG1 currents $\left(I_{\mathrm{hERG}}\right)$ in different cell types $(17,26)$. In neuroblastoma cells, this process is mediated by $\mathrm{G \alpha}_{\mathrm{i}}$ protein (17) and triggers integrin-dependent signaling cascades $(23,26)$. In addition, cell adhesion to fibronectin generally stimulates the formation of a macromolecular complex between hERG1 and $\beta_{1}$ integrin (hERG1/ $\beta_{1}$ integrin complex) (23), which may recruit growth factor receptors such as vascular endothelial growth factor receptor-1 (27) and CXCR-4 (28). Therefore, hERG1 takes part in multiprotein complexes that constitute major signaling centers in different cell types.

How hERG1 and $\beta_{1}$ integrin interact and how the ensuing macromolecular complex responds to diffusible signals is unknown. The role 
A
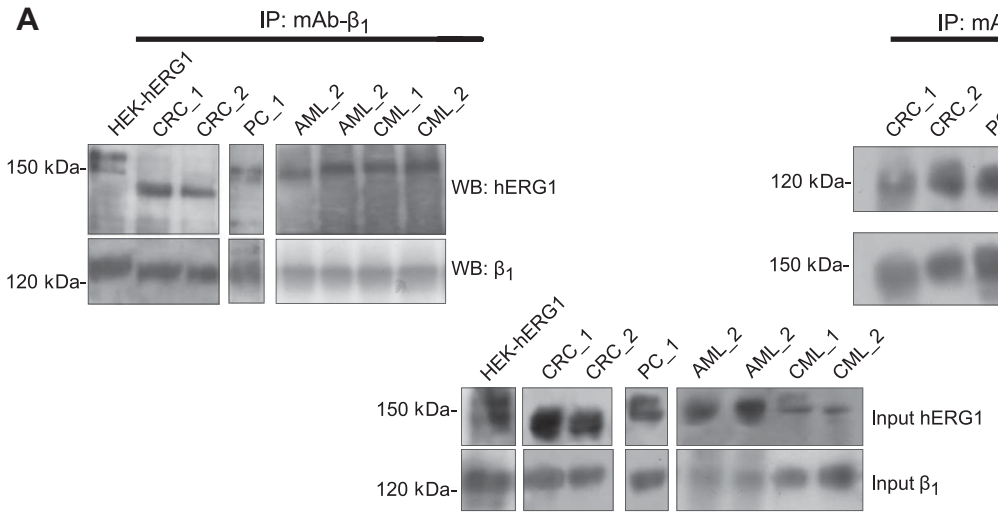

B

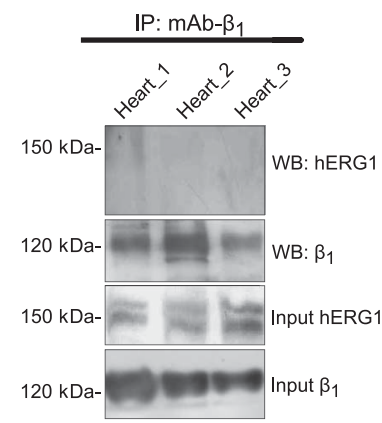

C

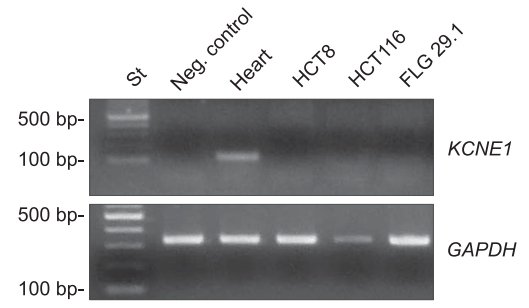

D

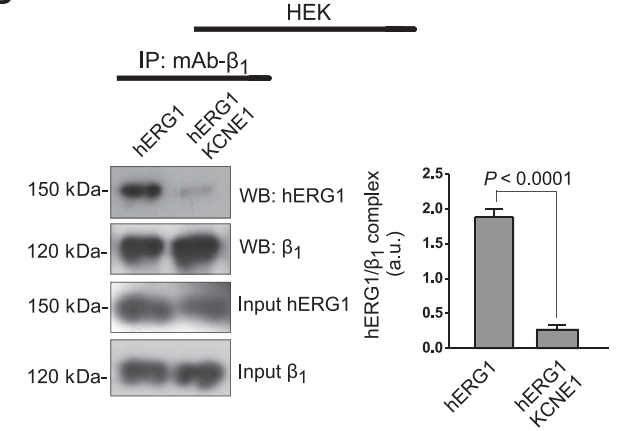

Input hERG1 $\beta_{1}$

E
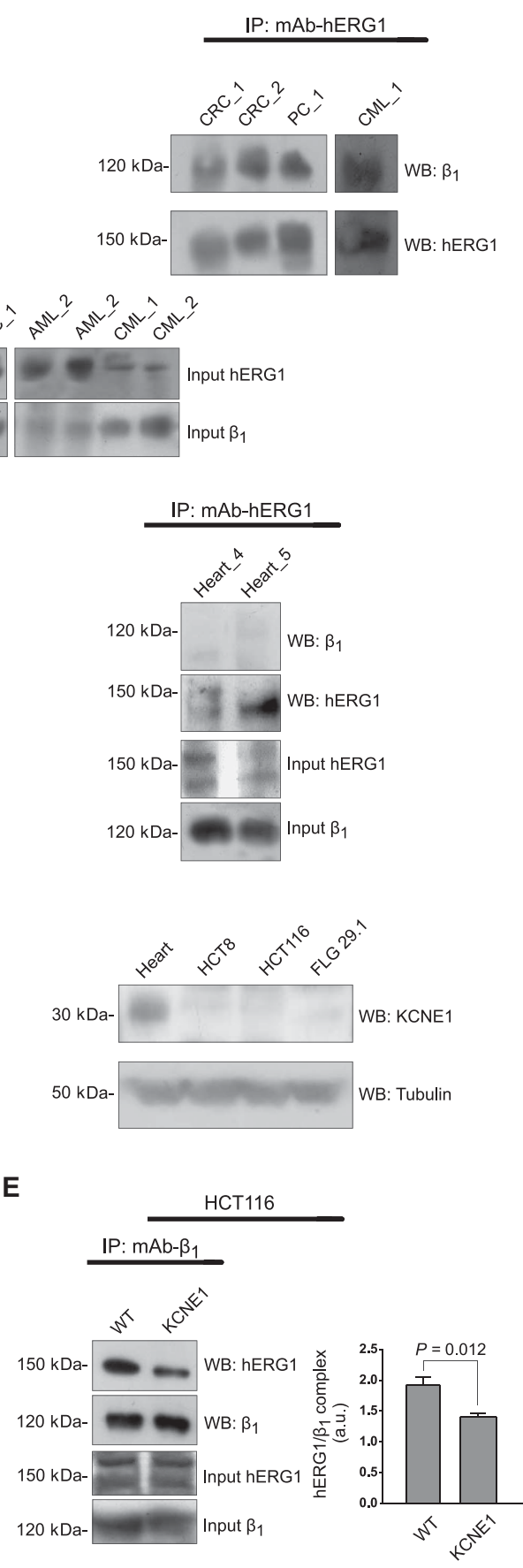

Fig. 1. hERG1 and $\beta_{1}$ integrin associate in human cancer tissue but not in cardiac tissue. (A) Left: Coimmunoprecipitation of $\beta_{1}$ and hERG1 from CRC, PC, AML, and CML samples. Right: Coimmunoprecipitation of hERG1 and $\beta_{1}$ from CRC, $\mathrm{PC}$, and one CML sample. Bottom: Western blots (WBs) of total lysates (input) from the same tumor samples for hERG1 or $\beta_{1}$ integrin $\left(\beta_{1}\right)$. Representative of three independent experiments. mAb, monoclonal antibody. (B) Coimmunoprecipitation of $\beta_{1}$ and hERG1 (left) and coimmunoprecipitation of hERG1 and $\beta_{1}$ in human heart atria. Representative of at least three independent experiments. Differences in the molecular masses of hERG1 bands in the various cancer tissues are due to differential posttranslational processing $(56,57)$. (C) Left: QPCR of KCNE1 and GAPDH in a representative heart sample, two CRC cell lines (HCT8 and HCT116), and one AML cell line (FLG 29.1). Lane 1: 100-base pair (bp) standard (St); lane 2: negative control (Neg. control). Representative of three independent experiments. Right: Western blot of total protein lysates from the same samples for KCNE1 and tubulin. (D and E) Coimmunoprecipitation of $\beta_{1}$ and hERG1 from HEK cells (D) or HCT116 cells (E) transfected or not with KCNE1. a.u., arbitrary units. Representative of three independent experiments performed in each cell line; the corresponding densitometric results are given in the bar graph. $P$ values were calculated with respect to HEK-hERG1 and HCT116-wild type (WT) cells, respectively; Student's $t$ test. of $\mathrm{K}^{+}$flow as compared to nonconducting hERG1-related signals is also unclear. Here, we first investigated why the $h E R G 1 / \beta_{1}$ integrin complex was formed in cancer cells but not in heart tissue, where hERG1 is found with its ancillary subunits (11). Next, we studied the mechanism of the interaction of the two proteins using deletion mutants. The protein-protein interaction was further confirmed with Förster resonance energy transfer (FRET) analysis (29-32). Using hERG1 mutants with alterations in either conductance or gating, we sought to discriminate between the contributions of the channel's conformational state and $\mathrm{K}^{+}$flow to the assembly of the macromolecular complex and to downstream signaling. Finally, we studied the in vivo effects of disrupting the interaction between hERG1 and $\beta_{1}$ integrin on the growth and metastasis of breast cancer cells xenografted in mice.

\section{RESULTS}

KCNE1 and $\beta_{1}$ integrin compete for binding to hERG1

We compared the interaction between hERG1 and $\beta_{1}$ integrin in primary cancer samples and surgical samples from human hearts. $\beta_{1}$ integrin immunoprecipitates from colorectal cancers (CRCs), pancreatic cancers (PCs), acute myeloid leukemia (AML), and chronic myeloid leukemia (CML) contained a hERG1 band (Fig. 1A, left). Conversely, a $\beta_{1}$ integrin band was detected in hERG1 immunoprecipitates from CRC, PC, and CML-1 samples (Fig. 1A, right). In contrast, no association between hERG1 and $\beta_{1}$ integrin was detected in cardiac tissue from human atria (Fig. 1B). Because hERG1 associates with KCNE1 in cardiomyocytes $(10,33)$, we hypothesized that the ancillary protein could impair the channel interaction with $\beta_{1}$ integrin. We first verified that both hERG1 (Fig. 1B, input hERG1) and KCNE1 (Fig. 1C) were present in the heart samples. In contrast, neither the KCNE1 transcript nor the corresponding protein was found in the tumor cell lines with a hERG1/ $\beta_{1}$ integrin complex (Fig. 1C) $(26,27)$. Next, we expressed KCNE1 in HCT116 cells, which have endogenous hERG1, or in human embryonic kidney (HEK) 293 cells, which lack the potassium channel and were transfected with hERG1. The presence of transcript for KCNE1 was verified by quantitative real-time polymerase chain reaction (QPCR) (fig. S1, A and B). In HEK cells plated on fibronectin, the 
formation of the hERG1/ $\beta_{1}$ integrin complex was inhibited by KCNE1 (Fig. 1D). Similar results were obtained in HCT116 cells (Fig. 1E), although the effect was smaller. These results suggested that KCNE1 and $\beta_{1}$ integrin competed for binding to hERG1, which could explain why formation of the hERG1/ $\beta_{1}$ integrin complex occurs only in neoplastic tissue.

A
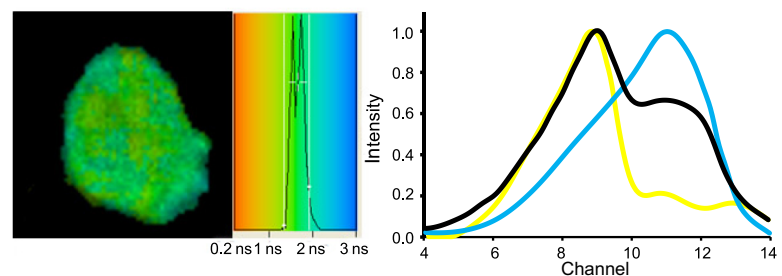

B

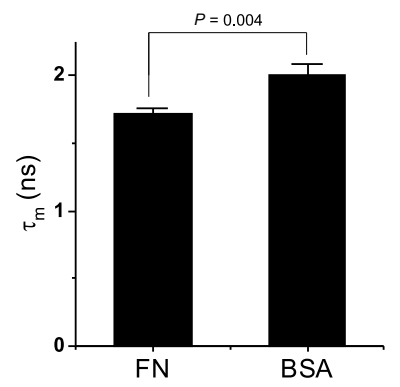

D
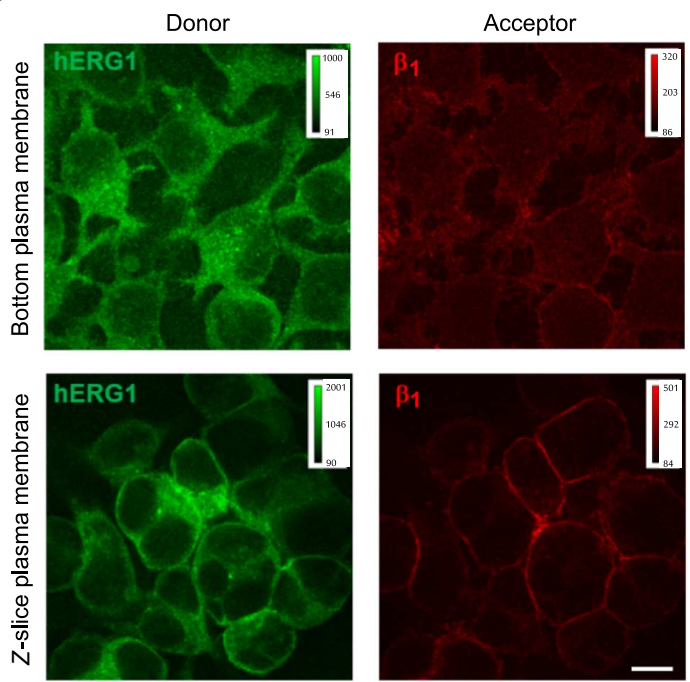

\section{hERG1 and $\beta_{1}$ integrin may directly physically interact}

To study how hERG1 and $\beta_{1}$ integrin assemble, we used HEK cells transfected with wild-type (HEK-hERG1) or mutant hERG1 complementary DNA (cDNA). Endogenous $\beta_{1}$ integrin is present in these cells and coimmunoprecipitated with hERG1 in cells allowed to adhere to fibronectin

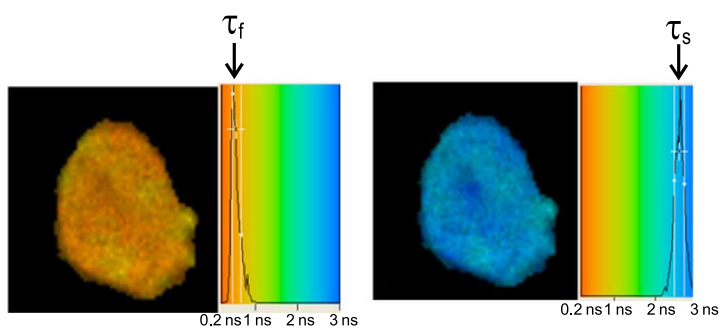

C

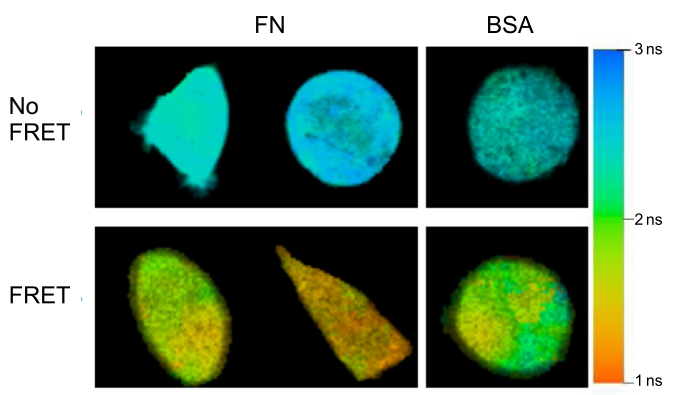

E

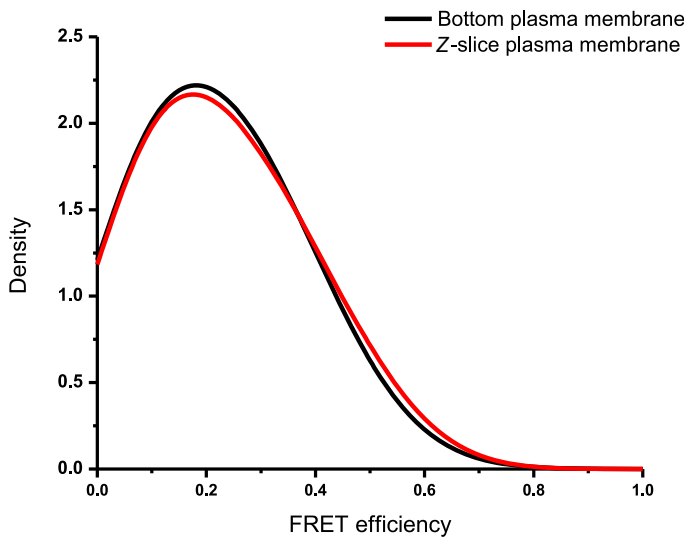

Fig. 2. FRET shows close interaction of $\mathbf{h E R G} 1$ and $\boldsymbol{\beta}_{\mathbf{1}}$ integrin on the plasma membrane. (A) Left: Image in false colors corresponding to the $\tau_{\mathrm{m}}$ shown in the lifetime histogram and emission spectra (black curve) for a cell showing FRET. These spectra are compared to the measured emission spectra for eCFP (cyan curve) and eYFP (yellow curve). Right: Image in false colors and related histogram corresponding to fast lifetime values $\tau_{f}$ (left) and to slow lifetime values $\tau_{s}$ (right) for the same cell. Images are representative of four independent experiments, in which 104 total cells were analyzed. (B) FRET measurements, expressed as donor mean lifetimes carried out on HEK-hERG1 cells seeded onto fibronectin (FN) ( $n=14$ cells, from three independent experiments) or BSA ( $n=12$ cells, from three independent experiments). $P$ value was calculated with respect to cells seeded on BSA; Student's $t$ test. (C) Examples of lifetime images for a cell on fibronectin not showing FRET (top left), for a cell on fibronectin showing FRET (bottom left), for a cell on BSA not showing FRET (top right), and for a cell on BSA showing FRET (bottom right). (D) Representative raw images of donor (hERG1, left) and acceptor ( $\beta_{1}$, right) (before acceptor photobleaching) at two different focal planes, namely, "Bottom plasma membrane" and "Z-slice plasma membrane" in HEK 293 cells. Scale bar, $10 \mu$ m. The intensity scale is displayed in the top right corner of each image. Each image is representative of 12 total images per experimental condition (WT and mutants). (E) hERG1/ $\beta_{1}$ FRET efficiency histograms of bottom plasma membrane (black solid line) images ( $n=6$ images, each sampling at least five cells obtained from two different transfection experiments) and $Z$-slice plasma membrane (blue solid line) images ( $n=6$ images, each sampling at least five cells obtained from two different transfection experiments), filtered for $\beta_{1}$ abundance (see the Supplementary Materials on image processing protocol). Mean FRET efficiencies were $0.23 \pm 0.05$ (Bottom plasma membrane) and $0.24 \pm 0.05$ ( $Z$-slice plasma membrane). The underlying distributions were not statistically different [two-sample Kolmogorov-Smirnov (K-S) test]. 
(fig. S2) (23). To study whether the two proteins interacted directly, we applied hyperspectral-fluorescence lifetime imaging microscopy-FRET (HS-FLIM-FRET) (34) on HEK cells transfected with cyan fluorescent protein (CFP)-labeled hERG1 and yellow fluorescent protein (YFP)labeled $\beta_{1}$ integrin (fig. S3). The fluorescence decay was characterized by biexponential behavior with one long-lived and one fast-decaying component. From each cell (Fig. 2A), we obtained the lifetimes $\left(\tau_{\mathrm{s}}\right.$

A
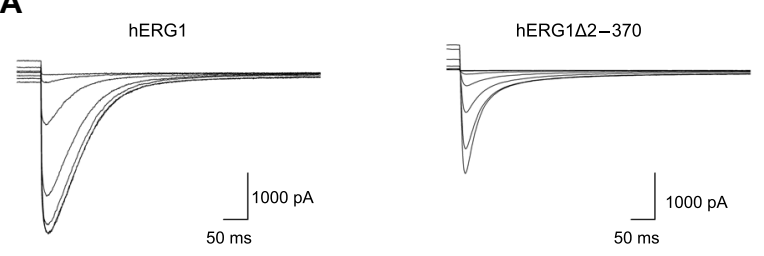

B
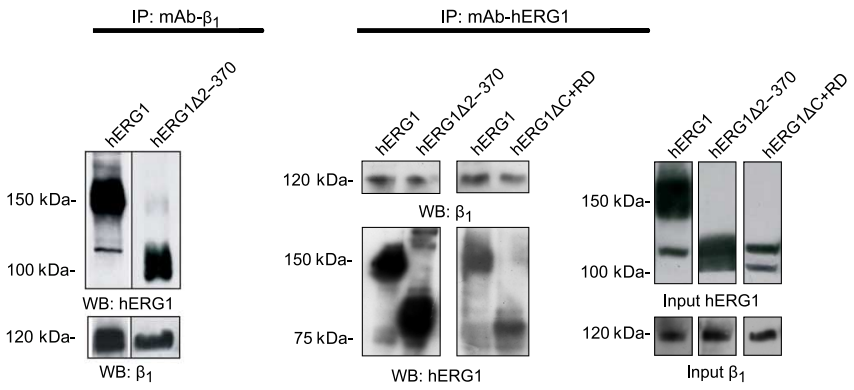

D
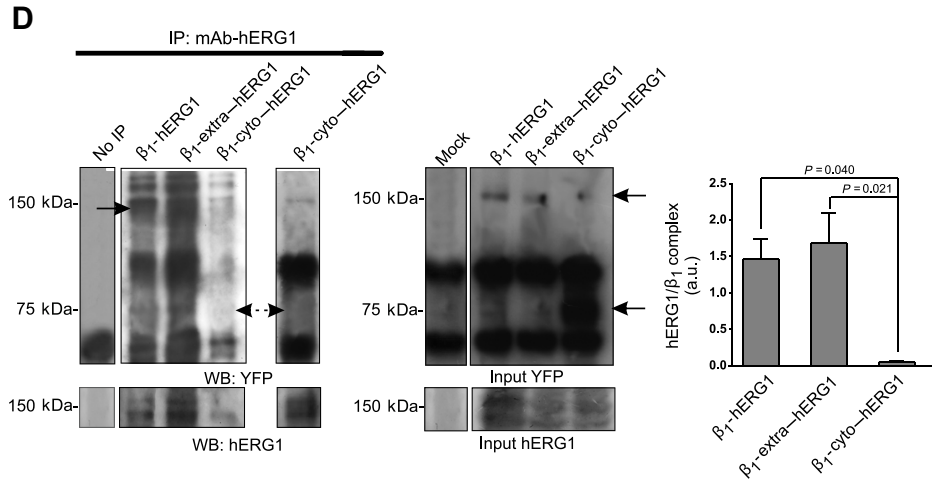

and $\tau_{\mathrm{f}}$, respectively) and fraction sizes ( $A_{\mathrm{s}}$ and $A_{\mathrm{f}}$, respectively) of both components for all pixels of the image (control experiments are shown in figs. S4 and S5). The slow lifetime was similar to the lifetime of enhanced CFP (eCFP) when no energy transfer occurred $\left(\tau_{\mathrm{s}}=2.48 \pm\right.$ $0.09 \mathrm{~ns}$; see the Supplementary Materials). The fast lifetime is a signature of energy transfer and the close proximity of hERG1 to $\beta_{1}$ integrin. By using eq. S2 (Supplementary Materials and Methods), the calculated FRET efficiency was $0.77 \pm 0.04$. We also compared the HS-FLIM-FRET signals in cells cultured in fibronectin or bovine serum albumin (BSA). Measurements were stopped at $100 \mathrm{~min}$ because after this time, cells cultured in BSA progressively detached from the substrate. The donor mean lifetime values $\left(\tau_{\mathrm{m}}\right)$ were calculated using eq. S3 (Supplementary Materials). For cells cultured in fibronectin, $\tau_{\mathrm{m}}$ was $1.71 \pm 0.05$, whereas for cells cultured in BSA, $\tau_{\mathrm{m}}$ was $2.00 \pm 0.08$ (Fig. 2B). Shorter lifetimes in cells cultured in fibronectin indicated higher FRET efficiency in elongated cells that strongly adhered to the substrate (Fig. 2C, FRET). In contrast, longer lifetimes, which indicated lower FRET efficiency or no FRET, corresponded to round cells or cells fixed to the substrate through small filopodia (Fig. 2C, No FRET).

\section{The hERG1/ $\boldsymbol{\beta}_{1}$ integrin complex is localized to the plasma membrane} To demonstrate that hERG 1 and $\beta_{1}$ integrin interaction occurred at the plasma membrane, the functional site of both proteins, we performed FRET experiments by acceptor photobleaching imaging (29) on fixed HEK cells. Donors and acceptors were labeled using monoclonal antibodies directed against hERG1, which was coupled to Alexa Fluor 488, and against $\beta_{1}$ integrin, which was coupled to Alexa Fluor 546 (figs. S6 and S7). Confocal imaging allowed us to image FRET at various locations in the cell. We chose two different focal planes, one located at the plasma membrane close to the fibronectin-coated substrate (Fig. 2D, Bottom plasma membrane) and one located at about half of the maximal cell thickness (Fig. 2D, Z-slice plasma membrane). Data analysis confirmed that the FRET signal originated on, or was proximal to, the plasma membrane (acceptor mask in fig. S7B). The average FRET efficiencies calculated for the bottom and the equatorial (Z-slice) plasma membranes were similar (Fig. 2E). Hence, the interaction between hERG1 and $\beta_{1}$ integrin was not restricted to the membrane portions involved in cell-substrate adhesion but also occurred in membrane patches distant from the 
adhesion sites. The mean FRET efficiency for both locations was $0.24 \pm$ 0.05 , about fourfold smaller than the value measured by HS-FLIMFRET, which we attribute to the different labeling methods for hERG1 and $\beta_{1}$ integrin.

\section{Interaction between $\mathrm{hERG} 1$ and $\beta_{1}$ integrin does not require the hERG1 intracellular domains or the cytoplasmic C-terminal domain of $\beta_{\mathbf{1}}$ integrin}

To define the molecular domains implicated in the complex formation, we performed electrophysiological analysis of HEK cells stably expressing forms of hERG1 lacking the entire $\mathrm{N}$ terminus (hERG1 $12-370$ ) or the $\mathrm{C}$ terminus (hERG $\Delta \mathrm{C}+\mathrm{RD}$ ) except for the amino acids from 1018 to 1122 , which constitute the recapitulation domain (RD) that allows hERG1 insertion into the plasma membrane (35). As expected, whole-cell current recordings indicated that hERG1 $\Delta 2-370$ displayed the typical fast deactivation conferred by $\mathrm{N}$ terminus deletion (36), whereas hERG1 $\Delta \mathrm{C}+\mathrm{RD}$ generally displayed current amplitudes considerably smaller than those of hERG1 (Fig. 3A) (35). Immunoprecipitation analysis of these cells suggested that the cytoplasmic hERG1 domains were dispensable for assembly with $\beta_{1}$ integrin (Fig. 3B). To test whether the intracellular domain of $\beta_{1}$ integrin interacted with hERG1, we transfected hERG1 and the following YFP-labeled constructs into GD25 cells, which are deficient in $\beta_{1}$ integrins (37): (i) full-length $\beta_{1}$ integrin, (ii) $\beta_{1}$ integrin lacking the $C$ terminus ( $\beta_{1}$-extra), and (iii) $\beta_{1}$ integrin $C$ terminus linked to the transmembrane and extracellular portions of the interleukin-2 receptor ( $\beta_{1}$-cyto) (Fig. 3, C and D). Coimmunoprecipitation analysis showed that hERG1 coimmunoprecipitated with $\beta_{1}$ integrin and $\beta_{1}$-extra but not with $\beta_{1}$-cyto (Fig. 3D), suggesting that the cytoplasmic domain of $\beta_{1}$ integrin was not necessary for interaction with hERG1. We confirmed this conclusion by performing coimmunoprecipitation using an antibody against $\beta_{1}$ integrin in GD25- $\beta_{1}$-TR cells, which stably express $\beta_{1}$ integrin lacking the $C$ terminus (Fig. $3 \mathrm{E}$ ). We concluded that the cytoplasmic domains of hERG1 and $\beta_{1}$ integrin were not necessary for the interaction of these proteins.
A

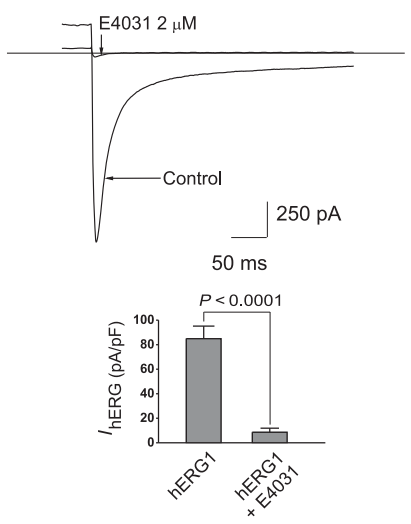

D
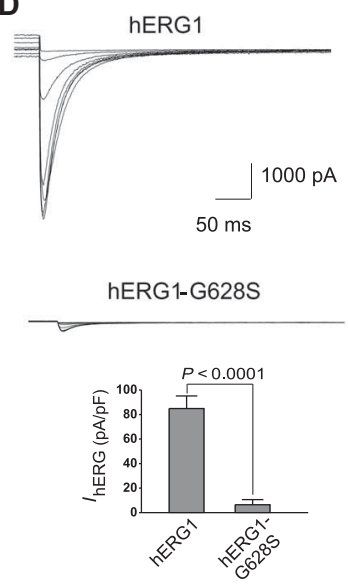

B

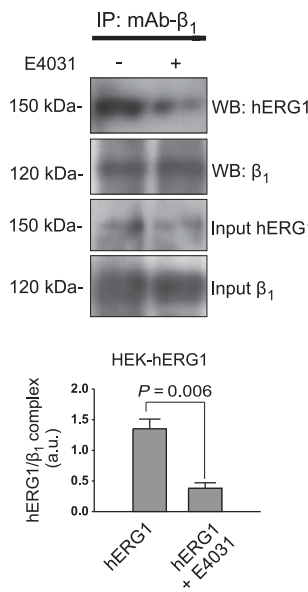

E
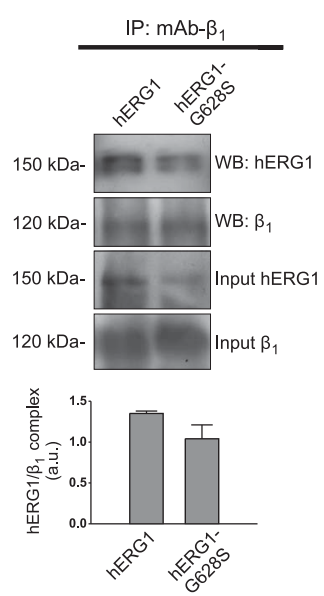

C

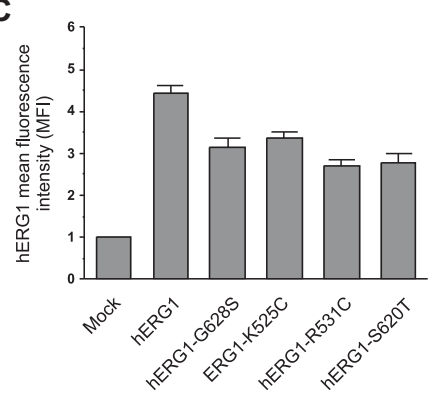

Fig. 4. Role of hERG1 current in the macromolecular complex formation and surface expression of WT and mutant hERG1 proteins. (A) Effect of E4031 on whole-cell WT hERG1 currents in HEK-hERG1 cells. Current traces show the blocking effect on tail currents at $-120 \mathrm{mV}$. Bars give the average hERG1 peak current densities in the presence or absence of $2 \mu \mathrm{M} \mathrm{E} 4031$ ( $n=5$ cells analyzed in three independent experiments). $P$ value was calculated with respect to hERG1 cells; Student's $t$ test. (B) Coimmunoprecipitation of $\beta_{1}$ and hERG1 from HEK-hERG1 cells seeded onto fibronectin, in the absence or presence of $40 \mu \mathrm{M}$ E4031. Representative of three independent experiments; the corresponding densitometric results are given in the bar graph. $P$ value was calculated with respect to hERG1 cells; Student's $t$ test.

(C) Membrane abundance of the indicated hERG1 constructs measured in HEK cells by flow cytometry and expressed as mean fluorescence intensity (MFI). The mean fluorescence intensity of mock-transfected HEK cells was set as 1 . Representative of four independent experiments. Not statistically different, Kruskal-Wallis ANOVA with Dwass, Steel, CritchlowFligner (DSCF)'s post hoc method. (D) Whole-cell hERG1 currents in HEK-hERG1 cells (upper traces) and HEK-hERG1 G628S cells (lower traces). Only the tail current at $-120 \mathrm{mV}$ is shown (conditioning potentials are given in Materials and Methods). Currents were measured in the presence of $40 \mathrm{mM}$ extracellular $\mathrm{K}^{+}$. Bars give the corresponding average peak current densities for hERG1 and hERG1-G628S ( $n=8$ cells analyzed in three independent experiments). $P$ value was calculated with respect to $h E R G 1$ cells; Student's $t$ test. (E) Coimmunoprecipitation of $\beta_{1}$ and hERG1 from HEK-hERG1 and HEK-hERG1 G628S cells seeded onto fibronectin. Representative of five independent experiments; the corresponding densitometric results are given in the bar graph. Not statistically different, Student's $t$ test.

hERG1 gating, but not conduction, regulates the complex formation

The results obtained with $\mathrm{hERG} 1 \triangle \mathrm{C}+\mathrm{RD}$

(Fig. 3, A and B) suggested that impairing channel conduction did not affect the association of hERG1 with $\beta_{1}$ integrin. To further investigate this issue, we tested the complex formation in the presence of E4031, which blocks hERG1 (Fig. 4A) by binding to the $\mathrm{Phe}^{656}$ residue that protrudes into the channel pore (38). E4031 inhibited the complex formation by about $80 \%$ (Fig. 4B). This result could be explained if $\mathrm{K}^{+}$flux is critical for the complex formation or if the presence of E4031 locks a substantial fraction of the channels in the open conformation, which may have a lower affinity for $\beta_{1}$ integrin. To better distinguish these mechanisms, we expressed several mutant constructs in HEK cells: the nonconducting hERG1-G628S (38), hERG1R531C, and hERG1-K525C, which are S4 domain mutants with altered activation (39), and the noninactivating hERG1S620T (40). Flow cytometry analysis (41) indicated that the plasma membrane abundance of the mutants was $\sim 25$ to $30 \%$ less compared to that of wild-type hERG1 (Fig. 4C). The strong decrease in $\mathrm{K}^{+}$flow for the hERG1-G628S mutant is not accompanied by gating alterations (38). hERG1G628S current amplitudes were comparable to those displayed by hERG1 in the presence of E4031 (Fig. 4D). Cells expressing hERG1G628S had a mean membrane voltage $\left(V_{\mathrm{m}}\right)$ under resting conditions $\left(V_{\text {rest }}\right)$ of $-36.9 \pm$ $1.9 \mathrm{mV}$, compared to $-48.4 \pm 2.3 \mathrm{mV}$ in cells expressing wild-type hERG1. Formation of the $h E R G 1 / \beta_{1}$ integrin complex was not significantly impaired by hERG1G628S (Fig. 4E). Thus, although blocking 
A
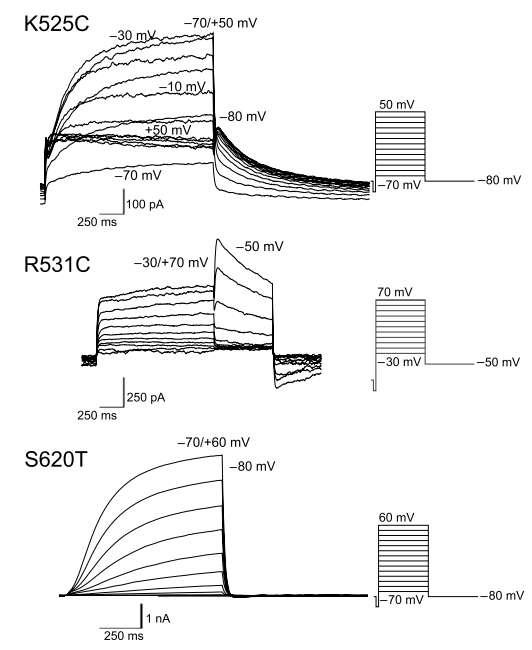

D
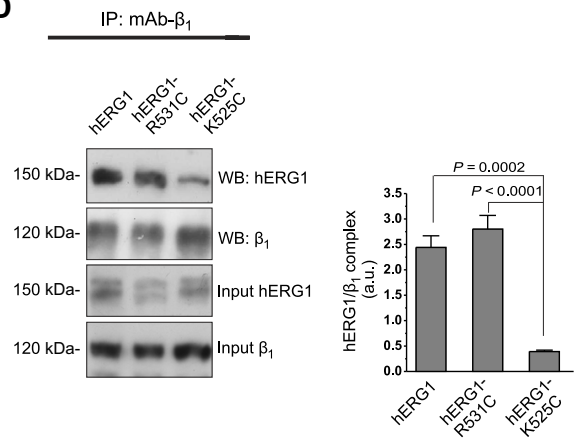

$\mathbf{F}$

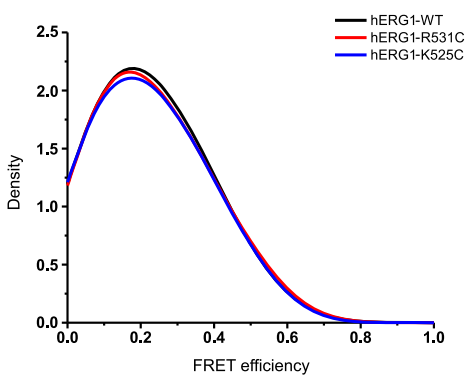

B

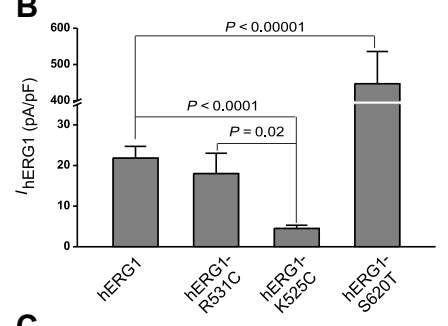

C

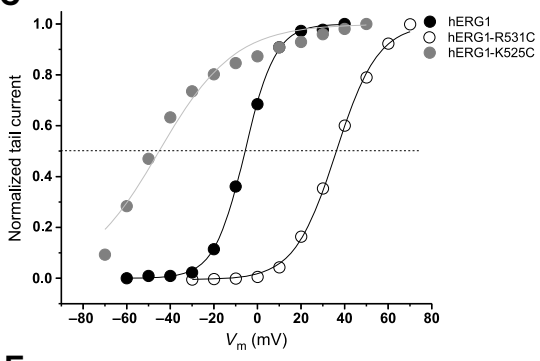

E

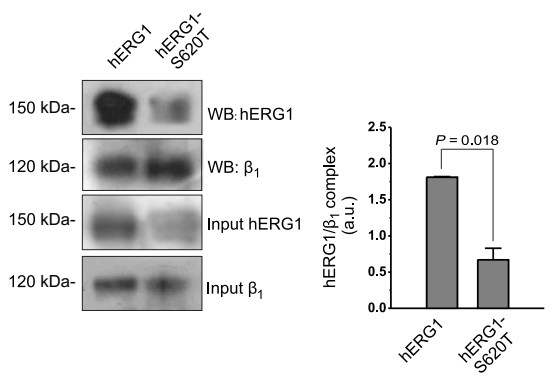

G

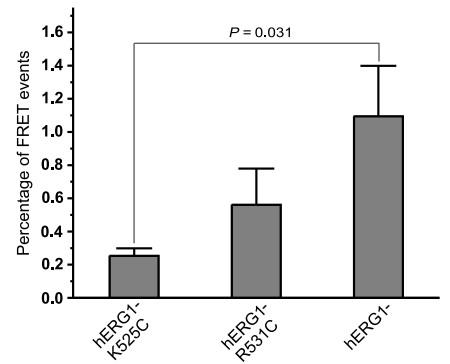

Fig. 5. hERG 1 gating regulates the $h E R G 1 / \beta_{1}$ complex formation. (A) Typical whole-cell current traces elicited in HEK cells stably transfected with either hERG1-K525C, hERG1-R531C, or hERG1-S620T. Representative of at least five cells per transfection condition analyzed in three independent experiments. (B) Comparison of the maximal current densities of hERG1-, hERG1-K525C-, hERG1-R531C-, and hERG1-S620T-expressing HEK cells. Data are average peak tail current densities calculated from at least five cells per transfection condition analyzed in three independent experiments, at test pulses of $+40,+50,+70$, and $+60 \mathrm{mV}$ for hERG1, hERG1-K525C, and hERG1-R531C. $P$ values were calculated with respect to hERG1 or hERG1-R531C cells; Kruskal-Wallis ANOVA with DSCF's post hoc method. (C) Activation curves of hERG1-, hERG1-K525C-, and hERG1-R531C-expressing HEK cells. Data points are normalized peak tail currents calculated from experiments as in (A). (D) Coimmunoprecipitation of $\beta_{1}$ and hERG1 from HEK 293 cells expressing hERG1, hERG1-K525C, and hERG1-R531C seeded onto fibronectin. Representative of three independent experiments; the corresponding densitometric results are given in the bar graph. $P$ values were calculated with respect to hERG1 cells; one-way ANOVA. (E) Same as (D) but for hERG1-S620T. Representative of three independent experiments; the corresponding densitometric results are given in the bar graph. $P$ value was calculated with respect to hERG1 cells; Student's $t$ test with Welch correction. (F) Probability distributions of FRET efficiency from confocal images of cells expressing $\beta_{1}$ integrin and hERG1, hERG1-R531C, or hERG1K525C ( $n=6$ images, each sampling at least five cells obtained from two different transfection experiments), filtered for $\beta_{1}$ abundance (see the Supplementary Materials for details on image processing). Mean FRET values are $0.24 \pm 0.06,0.23 \pm$ 0.06 , and $0.24 \pm 0.06$ for $h E R G 1$, hERG1-R531C, and hERG1-K525C, respectively. No statistical difference was found between all the distribution couples, as tested by two-sample K-S test $(P>0.05)$. (G) Percentage of FRET events for hERG1, hERG1R531C, and hERG1-K525C (assessing complex formation between hERG1 and $\beta_{1}$ ). $P$ value was calculated with respect to hERG1 cells; one-way ANOVA with Hochberg's (GT2) post hoc method.

channel conduction with E4031 impaired the complex assembly, data obtained with the nonconducting hERG1-G628S mutant suggested that decreased current flow per se was not the main determinant of the effect, in agreement with the results obtained with hERG1 $\Delta \mathrm{C}+\mathrm{RD}$.

To test the alternative hypothesis that the complex formation is sensitive to the channel's conformational state, we used mutants with different steady-state activation properties (Fig. 5A). Similar to wildtype hERG1, the hERG1-K525C protein was detected at the plasma membrane (Fig. 4C), although the maximal current density was lower (Fig. 5B) and the activation curve was shifted to a more negative $V_{\mathrm{m}}$ value (Fig. 5C). In agreement with previous work (39), the estimated $V_{1 / 2}$ of activation was around $-50 \mathrm{mV}$, and the measured $V_{\text {rest }}$ was $-59.1 \pm 1.1 \mathrm{mV}$. Hence, at steady state, hERG1-K525C channels spend more time in the open state than wild-type hERG1. hERG1-R531C had a similar maximal current density to that displayed by wild-type hERG1 (Fig. 5B). The estimated $V_{1 / 2}$ of activation was about $+35 \mathrm{mV}$ (Fig. $5 \mathrm{C}$ ). The corresponding $V_{\text {rest }}$ was $-42 \pm 2.6 \mathrm{mV}$, implying that a large fraction of the hERG1R531C channels resided in the closed state in our cells. Coimmunoprecipitation analysis showed that less $\beta_{1}$ integrin associated with hERG1-K525C than with wild-type hERG1 or hERG1-R531C (Fig. 5D). This result suggested that increasing the probability of the channel being in the open state decreased the complex formation. To further test this hypothesis, we used the noninactivating hERG1-S620T mutant, in which the openclosed transition is not complicated by the presence of the inactive state (40). Consistent with the absence of inactivation (Fig. 5A), hERG1-S620T had a maximal current density of $447 \pm 89 \mathrm{pA} / \mathrm{pF}$ (Fig. 5B), considerably higher than that measured for wild-type hERG1, and the measured $V_{\text {rest }}$ was $-59 \pm$ $2.3 \mathrm{mV}$. Similarly to hERG1-K525C, the complex association of hERG1-S620T with $\beta_{1}$ integrin was impaired compared to wildtype hERG1 (Fig. 5E). These results are consistent with the hypothesis that assembly of the hERG1 $/ \beta_{1}$ integrin complex was hindered when the probability of the channel transition toward the open state increased.

This mechanism was further investigated by FRET experiments in an acceptor photobleaching setting, on HEK cells expressing either wild-type hERG1, hERG1-R531C, or hERG1-K525C. The FRET efficiency 
distributions for the three conditions were not statistically different (Fig. $5 \mathrm{~F}$ ), demonstrating that energy transfer took place in both hERG1R531C- and hERG1-K525C-transfected cells. This finding suggested that the hERG1/ $\beta_{1}$ integrin complex, once it was formed, was structurally similar in wild-type and mutant channels. Next, we determined the percentage of FRET events, which reflected the abundance of hERG1/ $\beta_{1}$ integrin complexes. In agreement with the coimmunoprecipitation data, the complex formation was significantly impaired in cells expressing hERG1-K525C (Fig. 5G). These findings did not depend on the relative ratio between donor (hERG1) and acceptor ( $\beta_{1}$ integrin) present in the membrane (fig. S8).

\section{hERG1 current flow regulates FAK phosphorylation and cell proliferation in vivo}

We have previously shown that the $\beta_{1}$ integrin-dependent FAK phosphorylation in HEK cells expressing wild-type hERG1 relies on hERG1 activation (23). Hence, we studied how this process was modified by either E4031 or mutant channels. FAK was immunoprecipitated from HEK cells expressing wild-type hERG1 (treated or not treated with E4031) or one of the mutant hERG1 channels (Fig. 6A). The phosphorylation of $\mathrm{Tyr}^{397}$ in FAK (23) was impaired in cells expressing mutant hERG1 channels, with the exception of hERG1-S620T, compared to that in HEK cells expressing wild-type hERG1 (Fig. 6A). This finding suggests that the integrin-dependent FAK autophosphorylation required physiological hERG1 current amplitudes, which was impaired for different reasons by hERG1-G628S, E4031, hERG1-K525C, and hERG1-R531C, but not by hERG1-S620T. Because FAK controls various cellular functions, including cell survival and proliferation (42), by activating numerous signaling pathways, we tested the effects of the above treatments on in vivo cell growth. We subcutaneously injected HEK cells expressing wild-type hERG1, hERG1-G628S, hERG1K525C, or hERG1-R531C into immunodeficient nude mice. In mice that received cells expressing the different mutants or expressing wild-type hERG1 in the presence of E4031, the growth of subcutaneous masses was reduced compared to mice injected with HEK cells expressing wild-type hERG1 (Fig. 6B).

\section{Disrupting the $\mathrm{hERG} 1 / \boldsymbol{\beta}_{1}$ integrin complex inhibits metastasis of breast cancer cells}

We next tested whether such hERG1-mediated cell signals were present in cancer cells in which the abundance of hERG1 is abnormally high and hERG1 forms a macromolecular complex with $\beta_{1}$ integrin $(26,28)$. Specific hERG1 blockade with either Way 123,398 or E4031 impairs the phosphorylation of $\mathrm{Tyr}^{397}$ in $\mathrm{FAK}(23,26-28)$. This process was also studied in MDA-MB-231 breast cancer cells. Because these cells have low amounts of hERG1, we transfected our wild-type and mutant hERG1 constructs, which approximately doubled the membrane amount of hERG1 channels (Fig. 7A). Once again, only hERG1-K525C strongly impaired the $h E R G 1 / \beta_{1}$ integrin complex formation (Fig. $7 \mathrm{~B}$ ), whereas both hERG1-R531C and hERG1-K525C impaired the integrin-dependent phosphorylation of $\mathrm{Tyr}^{397}$ in FAK (Fig. 7C). We concluded that the signaling interaction between hERG1 and $\beta_{1}$ integrin was similar in HEK and cancer cells.

Finally, we studied the effects of disrupting the hERG1/ $\beta_{1}$ integrin complex in vivo, using MDA-MB-231 breast cancer cells transfected with either hERG1, hERG1-K525C, or hERG1-R531C. Cells were injected into either the right or left fourth breast of severe combined immunodeficient (SCID) mice. The number of breasts displaying tumor masses, the median volume of the tumor masses, and the presence of metastases in inguinal lymph nodes and lungs were determined 5 weeks after injection (Fig. 7D and fig. S9). Although the growth of tumors formed from MDA-MB-231 cells was not affected by overexpression of either mutant, the percentage of mice with metastases in either the inguinal lymph nodes or the lungs (Fig. 7, D and E) was significantly decreased when the cells expressed hERG1-K525C. The percentage of metastatic area (Fig. 7F) and the number of metastatic clusters (Fig. 7G) were decreased in mice injected with MDAMB-231 cells expressing hERG1-K525C, compared to those injected with cells expressing either wild-type hERG1 or hERG1-R531C. These results suggested that disrupting the $\mathrm{hERG} 1 / \beta_{1}$ complex by overexpressing the hERG1-K525C mutant impaired the metastatic process.

\section{DISCUSSION}

Fig. 6. Phosphorylation of $\mathrm{Tyr}^{397}$ in FAK is regulated by hERG1 current. (A) Phosphorylation (p) of $\mathrm{Tyr}^{397}$ in FAK in HEK cells expressing the indicated hERG1 constructs and treated or not treated with $40 \mu \mathrm{M}$ E4031, measured after cell seeding on fibronectin. Representative of four independent experiments; the corresponding densitometric results are given in the bar graph. $P$ values were calculated with respect to hERG1 cells; one-way ANOVA. hERG1-S620T versus hERG1 is not significant, Student's $t$ test. (B) Volume of tumor masses obtained in nude mice after subcutaneous injection of HEK 293 cells expressing the indicated hERG1 constructs. One group of mice injected with HEK-hERG1 cells was treated by intraperitoneal injection of E4031. Data are relative to six tumor masses for group (three mice per group). $P$ values were calculated with respect to mice injected with hERG1 cells; one-way ANOVA.
We used coimmunoprecipitation experiments and FRET imaging to examine whether hERG1 and $\beta_{1}$ integrin interacted directly on the plasma membrane. HSFLIM-FRET showed an increase in the fast lifetime component in cells seeded and spread onto fibronectin. The high FRET efficiency suggests that the two proteins were in close proximity, within the upper limit of the CFP-YFP dynamic range of $7.3 \mathrm{~nm}$ (43). Whether proximity was caused by direct interaction or through the participation of 
A

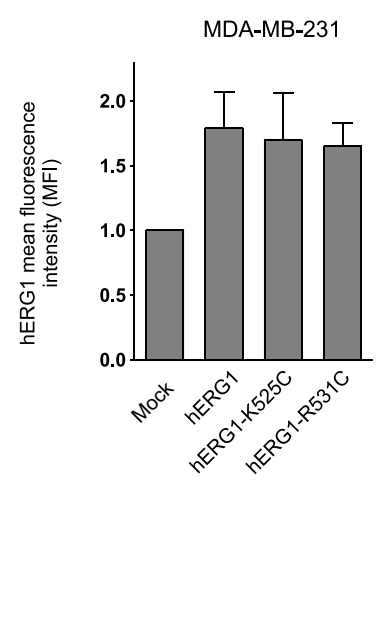

B
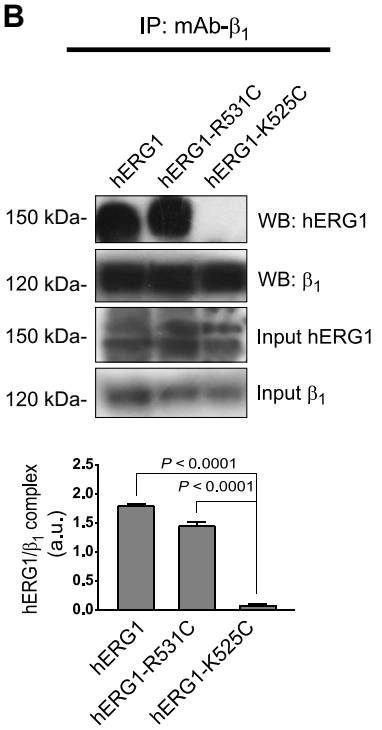

C
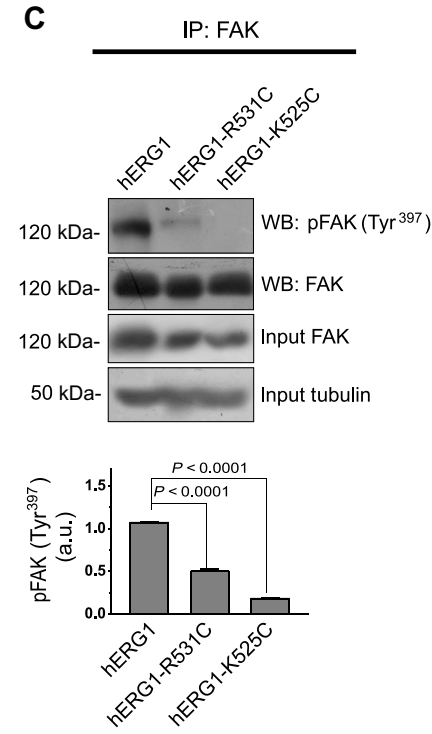

D

\begin{tabular}{|c|c|c|c|}
\hline MDA-MB-231 & hERG1 & hERG1-K525C & hERG1-R531C \\
\hline \multicolumn{4}{|l|}{ Local tumor growth } \\
\hline Number of tumor masses (\%) & 9/10 (90\%) & $10 / 10(100 \%)$ & 9/10 (90\%) \\
\hline Median tumor volume $\left(\mathrm{mm}^{3}\right)$ & $150(19-300)$ & $122(33-300)$ & $212(33-300)$ \\
\hline \multicolumn{4}{|l|}{ Metastases } \\
\hline $\begin{array}{l}\text { Inguinal lymph nodes } \\
\text { Number of mice with macroscopic } \\
\text { metastases (\%) }\end{array}$ & $2 / 5(40 \%)$ & $0 / 5(0 \%)$ & $3 / 5(60 \%)$ \\
\hline $\begin{array}{l}\text { Lung } \\
\text { Number of mice with macroscopic }\end{array}$ & $2 / 5(40 \%)$ & $0 / 5(0 \%)$ & $4 / 5(80 \%)$ \\
\hline
\end{tabular}

E
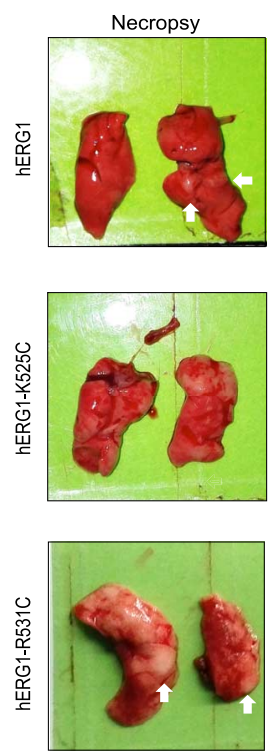

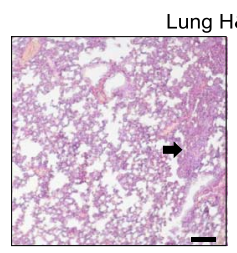

Lung H\&E
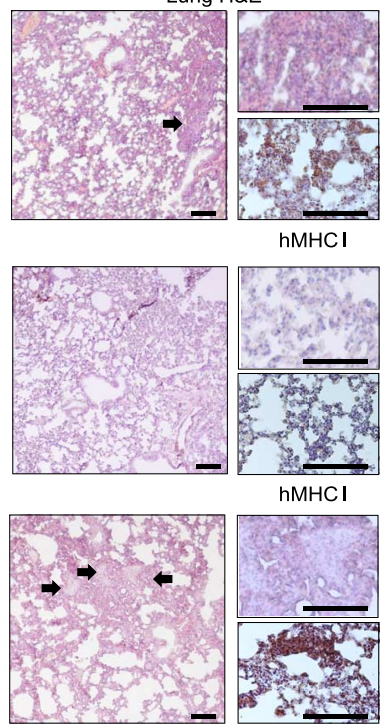

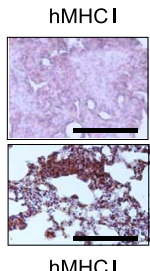

F

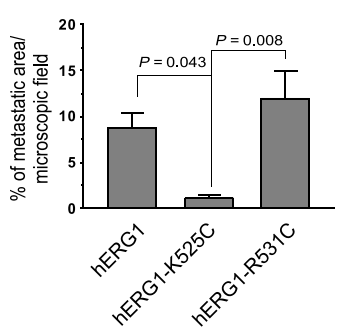

G

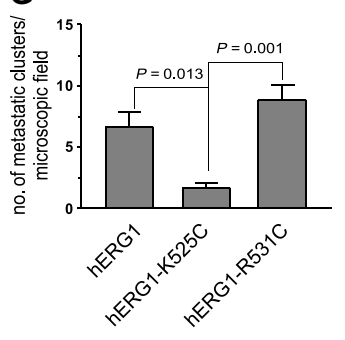

Fig. 7. Effects of inhibiting hERG1 currents and $h E R G 1 / \beta_{1}$ complex formation on neoplastic progression. (A) Expression of the indicated hERG1 constructs transfected in MDA-MB-231 cells, measured as in Fig. 4C. Bars show the average hERG1 mean fluorescence intensity of three independent experiments. Not statistically different, Kruskal-Wallis ANOVA with DSCF's post hoc method. (B) Coimmunoprecipitation of $\beta_{1}$ and hERG1 from MDA-MB-231 cells expressing the indicated $\mathrm{hERG} 1$ constructs seeded onto fibronectin. Representative of three independent experiments; the corresponding densitometric results are given in the bar graph. $P$ values were calculated with respect to hERG1 and hERG1-R531C cells; one-way ANOVA. (C) Phosphorylation of $\mathrm{Tyr}^{397}$ in FAK in MDA-MB-231 cells expressing the indicated hERG1 constructs. Representative of three independent experiments; the corresponding densitometric results are given in the bar graph. $P$ values were calculated with respect to hERG1 cells; one-way ANOVA. (D) Table summarizing quantitative data obtained from the breast orthotopic xenograft model. (E) Left (Necropsy): Images of lungs from representative animals in which MDA-MB-231 cells expressing the indicated hERG1 constructs were orthotopically injected in the breast. Middle (Lung H\&E): Lung sections labeled with H\&E (hematoxylin and eosin) from the same animal as in the left panels. Right: Magnified images and sections immunostained with an antibody against human major histocompatibility complex I (hMHC I). Scale bars, $100 \mu \mathrm{m}$. (F) Percentage of metastatic area and $(\mathbf{G})$ number of metastatic clusters per microscopic field in the lungs of mice injected with MDA-MB-231 cells expressing the indicated hERG1 constructs. Values are averages of measurements obtained in at least three different microscopic fields in both mouse lungs in five mice for each injected experimental group. $P$ values were calculated with respect to hERG1 and hERG1-R531C cells; one-way ANOVA.

interacted on the plasma membrane in a manner that did not require the cytoplasmic domains of these proteins. Nevertheless, because the hERG1 C terminus regulates channel gating, an indirect contribution to the complex formation cannot be ruled out. Although we did not provide evidence excluding the fact that the two proteins interact through their extracellular domains, we favor the conclusion that the interaction takes place largely through the transmembrane portions. First, it seems unlikely that the highly extended open conformation of the activated integrin (44) would interact

other proteins in a multiprotein complex cannot be distinguished. The interaction was increased by cell adhesion on fibronectin. FRET experiments further demonstrated that hERG1 and $\beta_{1}$ integrin interaction occurred only at the plasma membrane and not in cytosolic compartments. Moreover, coimmunoprecipitation experiments showed that hERG1 and $\beta_{1}$ integrin substantially with the very short extracellular domain of hERG1. Second, the interaction between the transmembrane domains would seem more consistent with the gating dependence of the complex formation and the competition between $\beta_{1}$ integrin and KCNE1. The transmembrane domain of $\beta_{1}$ integrin is a short $\alpha$ helix, which also undergoes profound 
conformational changes upon integrin engagement (45), and could interact with the hERG1 domains that constitute the voltage sensor (11).

Instead, we found that assembly of this macromolecular complex depended on the channel's conformational state. We derived this conclusion by comparing the degree of the complex formation of wild-type and mutant hERG1 channels. From the respective steady-state activation curves (Fig. 5, A and C) (39, 40), hERG1 and hERG1-R531C should primarily reside in the closed (deactivated) state, whereas hERG1-K525C and the noninactivating hERG1-S620T should spend more time in the open state. Because the latter two mutants associated poorly with $\beta_{1}$ integrin, we concluded that the complex formation was hindered when hERG1 was in the open state. Consistent with this notion, we observed impairment of the complex formation in the presence of E4031 (Fig. 4A), which occupies the channel pore and thus maintains hERG1 in the open conformational state (11). Our interpretation is consistent with the results obtained with the nonconducting hERG1-G628S mutant, which shows impaired current flow but a greater ability to interact with $\beta_{1}$ integrin than hERG1K525C, hERG1-S620T, or wild-type hERG1 inhibited by E4031.

On the other hand, most of the conditions we tested tended to block the phosphorylation of $\mathrm{Tyr}^{397}$ in FAK (namely, autophosphorylation), which we used as an early marker of integrin-dependent downstream signaling. The common outcome of our treatments was a decrease in current flow through hERG1. E4031 blocked the channel pore; hERG1G628S had a very low intrinsic channel conductance; hERG1-R531C had a very low open probability at the $V_{\text {rest }}$ typical of our cells; hERG1$\mathrm{K} 525 \mathrm{C}$, although generally active at $V_{\text {rest }}$, tended nonetheless to display a lower whole-cell current amplitude than wild-type hERG1. The exception was hERG1-S620T, which generated a higher whole-cell current amplitude than that of wild-type hERG1 (Fig. 5, A and B). Consistent with our interpretation, phosphorylation of $\mathrm{Tyr}^{397}$ in FAK in cells expressing this mutant was similar to that in cells expressing wild-type hERG1.

We showed that these mechanisms operated in cancer cells. Under all conditions in which $I_{\text {hERG1 }}$ was impaired, in vivo tumor growth was decreased, suggesting that channel activation regulated cell proliferation, possibly by controlling signaling pathways downstream of FAK activation $(42,46)$. In contrast, disrupting the $h E R G 1 / \beta_{1}$ integrin complex impaired the metastatic process of breast cancer cells. Therefore, the molecular association between hERG1 and $\beta_{1}$ integrin appears to modulate the intracellular machinery related to cancer cell migration and invasiveness. Another biologically relevant finding was that the $h E R G 1 / \beta_{1}$ integrin complex was not detected in cardiomyocytes, which suggests that the complex could be specific to tumor tissue. This difference could be caused by direct competition between $\beta_{1}$ integrin and the hERG1 ancillary subunit KCNE1 in cardiomyocytes (Fig. 1). The competition between KCNE1 and $\beta_{1}$ integrin for hERG1 binding suggests that integrins could substitute for KCNE1 as a binding partner in certain tissues. Nonetheless, current evidence does not exclude the possibility that KCNE1 also binds to $\beta_{1}$ integrin.

Our present working hypothesis is that $\beta_{1}$ integrin-dependent cell signaling leading to autophosphorylation of FAK is regulated by hERG1 activation and requires normal current flow through the ion channel, whereas the membrane macromolecular complex tends to recruit hERG1 channels residing in the nonconducting states. As suggested by single-cell analysis $(16,17)$, hERG1 reaches maximal stimulation within a few minutes of cell adhesion onto fibronectin. It is possible that hERG1 activation occurring early during cell adhesion has a role in stimulating FAK phosphorylation and the ensuing signaling pathways, whereas the late formation of the macromolecular complex progressively shifts the hERG1 channel population toward the nonconducting state. This process would turn off the early signaling cascade and favor late integrin-dependent cytoskeleton reorganization toward migration and invasion $(7,8,46)$. Hence, these results open the way for studies aimed at developing hERG1-related pharmacological compounds targeting different aspects of the neoplastic progression.

\section{MATERIALS AND METHODS \\ Chemicals and antibodies}

Unless otherwise indicated, chemicals and antibodies were purchased from Sigma-Aldrich. Cell lysis buffer for protein extraction was from Cell Signaling Technology (\#9803), and Protein A/G Plus-Agarose for immunoprecipitation was from Santa Cruz Biotechnology (sc-2003). The following antibodies were used for immunoprecipitation and Western blots, at the indicated concentrations: mAb-hERG1 (DT-331, Di.V.A.L. Toscana SRL): immunoprecipitation, $5 \mu \mathrm{g} / \mathrm{mg}$ protein; rabbit polyclonal antibody against hERG1 C terminus (hERG1 CT panpolyclonal antibody; DT-552, Di.V.A.L. Toscana SRL): Western blot, 1:1000; rabbit polyclonal antibody against $\beta_{1}$ integrin $C$ terminus (RM12, Immunological Sciences): Western blot, 1:1000; mAb- $\beta_{1}$ (TS2/ 16, BioLegend): immunoprecipitation, $5 \mu \mathrm{g} / \mathrm{mg}$ protein; rabbit polyclonal antibody against green fluorescent protein (GFP)/YFP (Ab290, Abcam): Western blot, 1:1000; rabbit polyclonal antibody against total FAK (SC-8312, Santa Cruz Biotechnology): immunoprecipitation, $1 \mu \mathrm{g} / \mathrm{mg}$ protein and Western blot, 1:1000; rabbit monoclonal antibody against total FAK (EP695Y, Abcam): immunoprecipitation, $6 \mu \mathrm{g} / \mathrm{mg}$ protein and Western blot, 1:1000; rabbit polyclonal antibody against $\mathrm{pTyr}^{397}$ FAK (BioSource International): Western blot, 1:1000; mouse monoclonal antibody against KCNE1 (Abcam): Western blot, 1:500; rabbit polyclonal antibody against human MHC class I (H-300; sc25619, Santa Cruz Biotechnology): immunohistochemistry, 1:100. The secondary antibodies used for Western blotting were anti-rabbit immunoglobulin G (IgG) peroxidase antibody (1:10,000; whole molecule, A0545) and anti-mouse IgG peroxidase antibody (1:5000; whole molecule, A4416). The hERG1 inhibitor E4031 was used as previously described (23) at a final concentration of $40 \mu \mathrm{M}$, except for the patch-clamp experiments shown in Fig. 4A, where E4031 was used at $2 \mu \mathrm{M}$.

\section{Plasmids}

To produce the peCFP-hERG1 plasmid, the full hERG1 cDNA sequence was subcloned from pcDNA3.1(+)hERG1 into peCFP-N3 plasmid. Before cloning, to maintain the correct frame between eCFP and hERG1, two nucleotides were inserted between a Hind III recognition site and the hERG1 start codon: (i) a 500-bp PCR fragment of hERG1 cDNA amplified from pcDNA3.1(+)hERG1 plasmid using the forward primer TAAGCTTGGATGCCGGTGCGGAGG, with a Hind III recognition site (bold) at the $5^{\prime}$ end of fragment and the hERG1 start codon (italics) preceded by a mismatch of two nucleotides (underscored), and (ii) the reverse primer GACCGCACCGACGACTCCCGGG. After denaturation, the DNA fragment was amplified using Platinum PCR SuperMix Taq polymerase (Invitrogen) for 25 cycles (denaturation for $30 \mathrm{~s}$ at $94^{\circ} \mathrm{C}$, annealing for $1 \mathrm{~min}$ at $50^{\circ} \mathrm{C}$, and extension for $1 \mathrm{~min}$ at $72^{\circ} \mathrm{C}$ ). After cloning into a pCR II cloning vector (Invitrogen), the amplified fragment was digested with Hind III and Bst XI restriction enzymes and inserted into pcDNA3.1(+)hERG1, to substitute the first 500 bp of hERG1 cDNA. The modified hERG1 cDNA was then subcloned into peCFPN3 after Hind III/Bam HI double digestion. The peCFP-hERG1 functionality was tested by Western blot and patch-clamp experiments. 
The clone pCDNAhERG1 $\Delta \mathrm{C}+\mathrm{RD}$ was generated according to Kupershmidt et al. (35). Briefly, a 315-bp PCR fragment, spanning nucleotides 3052 to 3366 of hERG1 cDNA, was amplified from the pCDNAhERG1 plasmid. The forward primer was GGACTCGAGCCCACCCCCAGCCTCCTCAACATCCC, with a Xho I recognition site (bold) inserted at the $5^{\prime}$ end. The reverse primer was CTATCTAGACTACGGGGGCAGCTCCTCACACGCCATG, with a stop codon TAG (italics) and an Xba I recognition site (bold) inserted at the $3^{\prime}$ end of the fragment. The amplified DNA, digested with Xho I and Xba I restriction enzymes, was inserted into Xho I/Xba I-digested pCDNAhERG1 plasmid, substituting the whole C-terminal domain (amino acids 699 to 1159). The obtained clones were characterized by restriction pattern analysis and sequenced to verify the conservation of reading frame.

Wild-type $\beta_{1}$ integrin (47), $\beta_{1}$ integrin lacking the $C$ terminus $\left(\beta_{1^{-}}\right.$ extra) (48), and the $C$ terminus of $\beta_{1}$ integrin linked to the transmembrane and extracellular part of the interleukin- 2 receptor $\left(\beta_{1}\right.$-cyto) were cloned into the YFP Venus (YFP)-N1 vector. To generate the pYFP-N1 vector, YFP cDNA was amplified with Bam HI and Not I from the pCS2Venus vector (49) and substituted to eGFP in the peGFP-N1 vector (Clontech). The peCE- $\beta_{1}$ plasmid containing the full-length $\beta_{1} \mathrm{~A}$ cDNA was cut with Hind III and SnaB I to obtain a fragment of around $2000 \mathrm{bp}$ and amplified (SnaB 1 and Bam HI) to generate a fragment of around $400 \mathrm{bp}$. The two fragments were cloned into the pYFP-N1 plasmid cut on Hind III/ Bam HI. The fragments corresponding to the $\beta_{1}$-extra and $\beta_{1}$-cyto constructs were amplified and cloned into the PYFP-N1 vector cut with Kpn I and Eco RI.

\section{Cell culture and transfection}

Cells were routinely cultured at $37^{\circ} \mathrm{C}$ with $5 \% \mathrm{CO}_{2}$ in either Dulbecco's modified Eagle's medium (DMEM) (Euroclone) (HEK 293-ICLC, GD25, and MDA-MB-231 cells) or RPMI (Euroclone) (HCT116 and REH cells) supplemented with $10 \%$ fetal bovine serum (Euroclone). HEK cells expressing wild-type hERG1 were prepared as previously described (23). To prepare the other stably transfected cell lines, transfection was carried out with Lipofectamine 2000 (Invitrogen). Selection and further cell culture were performed in complete culture medium supplemented with geneticin (G418, Invitrogen) at $0.8 \mathrm{mg} / \mathrm{ml}$ for HEK cells and $2.0 \mathrm{mg} / \mathrm{ml}$ for HCT116 and MDA-MB-231 cells. For HS-FLIM-FRET experiments, HEK cells were transiently transfected with both peCFP-hERG1 and peYFP- $\beta_{1}$ integrin plasmids. GD25 cells were transiently cotransfected with the different $\beta_{1}$ integrin-YFP constructs and hERG1. GD25- $\beta_{1}$-TR cells were described in (47).

\section{Preparation of cells for FRET, immunoprecipitation, and patch-clamp experiments}

Cells were harvested by detaching them with $5 \mathrm{mM}$ (10 mM for GD25 cells) EDTA in phosphate-buffered saline (PBS) and resuspended in DMEM plus heat-inactivated BSA $(250 \mu \mathrm{g} / \mathrm{ml}$; Fraction V, Euroclone). Next, cells were seeded onto dishes coated first with fibronectin $(100 \mu \mathrm{g} / \mathrm{ml}$ in serum-free medium, at $37^{\circ} \mathrm{C}$ for 1 hour) and then with BSA for 1 hour. FRET measurements started $15 \mathrm{~min}$ after cell seeding and continued at $37^{\circ} \mathrm{C}$ for at least $100 \mathrm{~min}$. When necessary (Fig. 2B), dishes coated with BSA alone were also used.

\section{RNA extraction, reverse transcription PCR, and QPCR}

RNA extraction and reverse transcription were performed as previously described (50). Amplification of KCNE1 was performed by QPCR with $2 \mu \mathrm{l}$ of cDNA derived from cell lines and human heart RNA (Ambion), using a commercially available master mix (Invitrogen).
The primer sequences for KCNE1 were 5'-TCCATTGGAGGAAGGCATTA-3' (forward primer) and 5' -CGCTGTGGTGTTAGACAGGA-3' (reverse primer). PCR was performed as follows: denaturation at $94^{\circ} \mathrm{C}$ for $2 \mathrm{~min} ; 35$ cycles at $94^{\circ} \mathrm{C}$ for $30 \mathrm{~s}$, at $55^{\circ} \mathrm{C}$ for $1 \mathrm{~min}$, and at $72^{\circ} \mathrm{C}$ for $30 \mathrm{~s}$; and final extension cycle at $72^{\circ} \mathrm{C}$ for $10 \mathrm{~min}$. The same primers were used for QPCR analysis on HEK and HCT116 cells transfected with the KCNE1 plasmid. The SYBR Green fluorescent dye (Power SYBR Green PCR Master Mix, Applied Biosystems) method was applied. Glyceraldehyde-3-phosphate dehydrogenase gene was used as a standard reference, as in (26). Nontransfected cell lines were used for calibration.

\section{Immunoprecipitation and immunoblotting}

Procedures on cell lines were performed as previously described $(23,26)$. Cardiac and cancer tissues were obtained from individual donors undergoing surgery. Leukemia samples were obtained from bone marrow aspirates of patients at the onset of the disease. Patients were treated at the Molinette Hospital (Turin, Italy), at the Department of General Surgery of the Careggi Hospital (Florence, Italy), and at the Department of Hematology of the Careggi Hospital. Tissues were collected after informed written consent and after approval of the local ethics committee. All procedures were carried out at $4^{\circ} \mathrm{C}$. Samples were homogenized in cold protein extraction buffer $(1 \times$ cell lysis buffer) and sonicated for $30 \mathrm{~min}$. For immunoprecipitation, total lysates $(1.5 \mathrm{mg}$ for cell line extracts and $0.5 \mathrm{mg}$ for primary samples) were subjected to a preclearing step by incubating them with Protein A/G Plus-Agarose for 2 hours at $4^{\circ} \mathrm{C}$. Thereafter, cell lysates were immunoprecipitated with the appropriate antibody at the concentrations indicated in "Chemicals and antibodies." Coimmunoprecipitation of $\beta_{1}$ integrin and hERG1 lysates was performed with $\mathrm{mAb}-\beta_{1}$. Coimmunoprecipitation of hERG1 and $\beta_{1}$ integrin lysates was performed with mAb-hERG1. For both reactions, Western blotting was performed on immunoprecipitates and total lysates (input) from the same sample with polyclonal antibodies against hERG1 or $\beta_{1}$. To determine phosphorylation of $\mathrm{Tyr}^{397}$ in FAK, FAK was immunoprecipitated with a polyclonal antibody. Western blotting was performed on immunoprecipitates and total lysates with polyclonal antibodies against $\mathrm{pTyr}^{397}$ FAK, total FAK, or tubulin.

\section{Densitometric analysis}

Densitometric analysis was performed using ImageJ software (Image) 1.38, U.S. National Institutes of Health) on two different scans, after background subtraction, from at least three different experiments. When quantifying variations in hERG1- $\beta_{1}$ integrin interactions, the signal for the coimmunoprecipitated protein (for example, hERG1 when immunoprecipitating the $\beta_{1}$ integrin, or $\beta_{1}$ integrin when immunoprecipitating hERG1) was first divided by the signal of the protein used for immunoprecipitation (for example, $\beta_{1}$ integrin or hERG1) and then normalized to the signal of the corresponding protein in the total lysate (input hERG1 and input $\beta_{1}$ ). The resulting value is indicated as "hERG1/ $\beta_{1}$ integrin complex" throughout the manuscript and in the figures. FAK phosphorylation was measured in immunoprecipitates with FAK antibodies, first dividing the signal for $\mathrm{Tyr}^{397}$ of FAK by the signal of the immunoprecipitated FAK and then normalizing this value to the amount of FAK in the total lysate (input FAK).

\section{Flow cytometry}

Expression of wild-type and mutant hERG1 was assessed by a FACSCanto flow cytometer, as in (28). Acquisition and analysis were performed using FACSDiva software (BD Biosciences). Values are 
expressed as mean fluorescence intensity fold changes compared to nontransfected HEK or MDA-MB-231 mock-transfected cells.

\section{HS-FLIM-FRET experiments}

We used a multiphoton microscope consisting of a Nikon TE2000-U inverted optical microscope, equipped with a Nikon PCM2000 confocal laser scanning unit, modified to allow the use of an ultrafast laser source, equipped with a dichroic short-pass filter, suitable for multiphoton operation (650DCSPXR, Chroma Inc.). This system is made up of a mode-locked Ti:sapphire oscillator (Mira 900 F, Coherent Inc.) pumped by a frequency-doubled Nd:YVO4 laser system at $532 \mathrm{~nm}$ and $5 \mathrm{~W}$ (Verdi V5, Coherent Inc.). Emission is tunable in the range 700 to $980 \mathrm{~nm}$, with a typical pulse duration of $130 \mathrm{fs}$ and a repetition rate of $76 \mathrm{MHz}$. The laser beam, after passing through a Faraday isolator (to prevent a part of the laser beam returning to the laser cavity), was directly coupled to the confocal scanning unit. The confocal head is supplied with two output fluorescence emission channels (51). For the measurements, the light emitted from the sample was sent to a spectrograph, coupled to a fast 16-channel photon-counting photomultiplier, with a typical transit time spread of 150 ps (PML-16C, Becker \& Hickl GmbH). A two-photon cutoff filter (BG39, SCHOTT $\mathrm{GmbH}$ ) was inserted in the fluorescence path to remove unwanted back reflection of the laser light. Acquired signals were processed by the FLIM apparatus, which is based on time-correlated single-photon counting and allows the measurement of the fluorescence decay curve for each image pixel. Its core element is an SPC-830 module (Becker \& $\mathrm{Hickl} \mathrm{GmbH}$ ) that can measure the arrival time delays of individual photons with respect to a synchronization pulse train, provided by the signal of a fast photodiode (PHD-100, Becker \& Hickl GmbH) that detects a laser light reflection from the neutral density filter. Curve fitting with exponential decay curves to the measured data and displays of the results was performed by the software SPCImage (Becker \& Hickl $\mathrm{GmbH})$.

The specificity and sensitivity of the FLIM technique to detect FRET in cells were tested using HEK transiently cotransfected with $\beta_{1}$ integrin-eYFP and hERG1-eCFP, either as single or as double transfectants (fig. S3). The theoretical expressions used are described in Supplementary Materials and Methods ("Analysis of HS-FLIM-FRET data"). FLIM measurements can be affected by $\mathrm{pH}$, temperature, previous prolonged exposure to radiation, and ion concentration. Spectral control is thus essential, especially when performing FLIM experiments in living cells. The details of our procedure are given in Supplementary Materials and Methods ("Analysis of HS-FLIM-FRET data") and the legends to figs. S4 and S5.

\section{FRET by acceptor photobleaching experiments}

We used a custom-made confocal microscope equipped with a spinning disk unit, as previously described (52) and further detailed in the Supplementary Materials. FRET was detected by an increase of the donor signal in acceptor bleaching. From the ratio of the donor fluorescent emissions before and after acceptor photobleaching, the pixel-by-pixel value of FRET transfer was determined. The percentage of FRET events, which reflected the abundance of hERG $1 / \beta_{1}$ integrin complexes, was estimated as the number of pixels for which FRET was detected in relation to the number of pixels in which the $\beta_{1}$ integrin signal was simultaneously recorded. A detailed description of data acquisition and analysis is also reported in the Supplementary Materials, in which flowcharts are included for image acquisition and processing steps. Data analysis was performed using MATLAB (MathWorks). Experiments were performed on HEK cells expressing wild-type hERG1, hERG1R531C, or hERG1-K525C cultured for $45 \mathrm{~min}$ on fibronectin-coated slides. Cells were fixed with $4 \%$ paraformaldehyde and labeled with Alexa-conjugated mAb-hERG1 and mAb- $\beta_{1}$. Purified mAb-hERG1 and $\mathrm{mAb}-\beta_{1}$ were labeled using the Alexa Fluor 488 and 546 Protein Labeling Kits (Molecular Probes), respectively. Quantitative estimation of labeled antibodies was determined by electrophoresis in agarose gel and Coomassie Brilliant Blue staining.

\section{Patch-clamp recording}

$I_{\text {hERG1 }}$ was recorded in the whole-cell configuration of the patch-clamp technique, at room temperature $\left(25^{\circ} \mathrm{C}\right)$, with a MultiClamp $700 \mathrm{~A}$ amplifier (Molecular Devices), as previously described (53). E4031 was used at $2 \mu \mathrm{M}$. Background currents and leakage were measured in the presence of E4031 and subtracted from the total current. Unless otherwise indicated, $\left[\mathrm{K}^{+}\right]_{\mathrm{o}}$ was $5 \mathrm{mM}$. hERG1-K525C currents were recorded in $2 \mathrm{mM}$ extracellular $\left[\mathrm{K}^{+}\right]$. The activation curves for hERG1, hERG1 $\Delta 2-370$, hERG1 $\Delta \mathrm{C}+\mathrm{RD}$, and hERG1-G628S were determined from peak tail currents $\left(I_{\text {tail }}\right)$ at $-120 \mathrm{mV}$ (for $1.1 \mathrm{~s}$ ), after 15-s conditioning potentials from 0 to $-70 \mathrm{mV}$ (10-mV steps, spaced $4 \mathrm{~s}$ apart). The holding potential $\left(V_{\mathrm{H}}\right)$ was $0 \mathrm{mV}$. The activation curves for wild-type hERG1, hERG1-K525C, and hERG1-R531C were obtained after a stimulation protocol similar to that used by Zhang et al. (39). From a negative $V_{\mathrm{H}}$ $(-80$ to $-120 \mathrm{mV})$, we applied 1 -s test voltages (10-mV increments) every $15 \mathrm{~s}$. Test voltages $\left(V_{\mathrm{t}}\right)$ varied from -60 to $+40 \mathrm{mV}$ (hERG1), from -70 to $+50 \mathrm{mV}$ (hERG1-K525C), and from -30 to $+70 \mathrm{mV}$ (hERG1-R531C). $I_{\text {tail }}$ was elicited by repolarization to $-50 \mathrm{mV}$ ( $-80 \mathrm{mV}$ for hERG1$\mathrm{K} 525 \mathrm{C}$ ). The peak $I_{\text {tail }}$ obtained at each $V_{\mathrm{t}}$ was normalized to the maximum $I_{\text {tail }}\left(I_{\max }\right)$. The relation between $I_{\text {tail }} / I_{\max }$ and $V_{\mathrm{t}}$ was fit to a Boltzmann function with OriginPro 2015 (OriginLab) software.

\section{In vivo experiments}

Experiments were performed at the Animal House of the University of Florence (CESAL). Mice were housed in filter-top cages with a 12-hour dark-light cycle and had unlimited access to food and water. Procedures were conducted according to the laws for experiments on live animals (Directive 2010/63/EU) and approved by the Italian Ministry of Health (1279/2015-PR).

For subcutaneous xenografts, female nude mice (Harlan Laboratories) aged 5 to 6 weeks were injected subcutaneously in either flanks with $2 \times 10^{6}$ cells, resuspended in $50 \mu \mathrm{l}$ of ice-cold PBS and gently mixed with the same volume of ice-cold Matrigel. The following human cell lines were used: HEK-hERG1, HEK-hERG1-G628S, HEK-hERG1R531C, and HEK-hERG1-K525C. Each experimental group comprised three mice. One group of mice injected with HEK-hERG1 was treated daily with E4031 (20 mg/kg), injected into the peritoneum, for 2 weeks, starting the day after inoculum. The volume of tumor masses measured at the sacrifice ( 6 weeks after inoculation) was calculated by applying the ellipsoid equation.

For breast orthotopic xenografts, female SCID mice (Harlan Laboratories) aged 6 weeks were injected in both fourth mammary fat pads (five mice per group), with different cell types $\left(2 \times 10^{6}\right.$ cells per mouse): MDA-MB-231-hERG1, MDA-MB-231-hERG1-R531C, or MDAMB-231-hERG1-K525C. After injection, mice were monitored daily to ensure that they did not show any signs of suffering or disease (such as weight loss, abdominal distension, or impaired movement). Five weeks after injection, mice were euthanized, and tumor masses and tissues were collected and processed for histological analysis. The volume of tumor masses at the sacrifice was calculated by applying the following 
expression: $0.52 \times l_{\min }{ }^{2} \times l_{\max }$, where $l_{\min }$ and $l_{\max }$ are the minimum and maximum measured length of the tumor mass, respectively. Hematoxylin and eosin staining and immunostaining of lung metastases were performed as in (26). To quantify the metastatic burden, images were acquired on a Leica DM4000B microscope with a Leica DFC320 camera using Leica QWin software (Leica Microsystems). The percentage of metastatic areas per microscopic field was determined as in (54). At least three fields per mouse lung and both lungs were examined.

\section{Statistical analysis}

Unless otherwise indicated, data are given as mean values \pm SEM, with $n$ indicating the number of independent experiments. Statistical comparisons were performed with OriginPro 2015 and SAS 9.2 (SAS Institute) software. The normality of data distribution was checked with K-S test. In the case of normal distributions, each data set was first checked for variance homogeneity, using the $F$ test for equality of two variances and the Brown-Forsythe test for multiple comparisons. For data with unequal variances, the Welch correction was applied. For comparisons between two groups of data, we used the Student's $t$ test. A two-sample $\mathrm{K}-\mathrm{S}$ test was performed to test whether two underlying probability distributions differed. For multiple comparisons, one-way ANOVA followed by Bonferroni's post hoc test was performed to derive $P$ values. As reported in the figure legends, in case of unequal variances, ANOVA was followed by the Hochberg's GT2 post hoc method. In the case of nonnormal distributions, nonparametric Kruskal-Wallis ANOVA followed by DSCF's post hoc method was applied. The relevant $P$ values are reported in the figure panels and legends.

\section{SUPPLEMENTARY MATERIALS}

\section{www.sciencesignaling.org/cgi/content/full/10/473/eaaf3236/DC1}

Materials and Methods

Fig. S1. Characterization of HEK 293 and HCT116 cells transfected with KCNE1.

Fig. S2. Coimmunoprecipitation of HEK-hERG1 cells seeded on fibronectin.

Fig. S3. Characterization of HEK CFPhERG $1+\beta_{1}$ YFP cells.

Fig. S4. Fluorescence decay of a donor control sample.

Fig. S5. Control experiments to validate FRET results.

Fig. S6. Protocol for image acquisition of HEK 293 cells.

Fig. S7. Background correction and FRET map calculation.

Fig. S8. FRET efficiency as a function of the ratio of donor to acceptor.

Fig. S9. Lymph node metastases in mice orthotopically injected with MDA-MB-231-hERG1 and MDA-MB-231-hERG1-R531C cells.

References (57-65)

\section{REFERENCES AND NOTES}

1. A. Becchetti, S. Pillozzi, R. Morini, E. Nesti, A. Arcangeli, New insights into the regulation of ion channels by integrins. Int. Rev. Cell Mol. Biol. 279, 135-190 (2010).

2. A. Schwab, A. Fabian, P. J. Hanley, C. Stock, Role of ion channels and transporters in cell migration. Physiol. Rev. 92, 1865-1913 (2012).

3. A. Arcangeli, A. Becchetti, Complex functional interaction between integrin receptors and ion channels. Trends Cell Biol. 16, 631-639 (2006).

4. A. Arcangeli, lon channels and transporters in cancer. 3. Ion channels in the tumor cellmicroenvironment cross talk. Am. J. Physiol. Cell Physiol. 301, C762-C771 (2011).

5. R. O. Hynes, Integrins: Bidirectional, allosteric signaling machines. Cell 110, 673-687 (2002).

6. M. A. Arnaout, B. Mahalingam, J.-P. Xiong, Integrin structure, allostery, and bidirectional signaling. Annu. Rev. Cell Dev. Biol. 21, 381-410 (2005).

7. S. Cabodi, M. del Pilar Camacho-Leal, P. Di Stefano, P. Defilippi, Integrin signaling adaptors: Not only figurants in the cancer story. Nat. Rev. Cancer 10, 858-870 (2010).

8. E. M. Morse, N. N. Brahme, D. A. Calderwood, Integrin cytoplasmic tail interactions. Biochemistry 53, 810-820 (2014).

9. D. S. Harburger, D. A. Calderwood, Integrin signalling at a glance. J. Cell Sci. 122, 159-163 (2009).

10. J. Mitcheson, A. Arcangeli, The therapeutic potential of $h E R G 1 \mathrm{~K}^{+}$channels for treating cancer and cardiac arrhythmias, in Ion Channel Drug Discovery, B. Cox, M. Gosling, Eds. (RSC Publishing, 2015), vol. 39, pp. 258-296.
11. J. I. Vandenberg, M. D. Perry, M. J. Perrin, S. A. Mann, Y. Ke, A. P. Hill, hERG K ${ }^{+}$channels: Structure, function, and clinical significance. Physiol. Rev. 92, 1393-1478 (2012).

12. A. Arcangeli, A. Becchetti, Novel perspectives in cancer therapy: Targeting ion channels. Drug Res. Updat. 21-22, 11-19 (2015).

13. A. Arcangeli, O. Crociani, E. Lastraioli, A. Masi, S. Pillozzi, A. Becchetti, Targeting ion channels in cancer: A novel frontier in antineoplastic therapy. Curr. Med. Chem. 16, 66-93 (2009).

14. J. Jehle, P. A. Schweizer, H. A. Katus, D. Thomas, Novel roles for hERG $\mathrm{K}^{+}$channels in cell proliferation and apoptosis. Cell Death Dis. 2, e193 (2011).

15. A. Arcangeli, O. Crociani, L. Bencini, Interaction of tumor cells with their microenvironment: lon channels and cell adhesion molecules. A focus on pancreatic cancer. Philos. Trans. R. Soc. London Ser. B 369, 20130101 (2014).

16. A. Becchetti, A. Arcangeli, M. R. Del Bene, M. Olivotto, E. Wanke, Response to fibronectinintegrin interaction in leukemia cells: Delayed enhancing of a $\mathrm{K}^{+}$current. Proc. Biol. Sci. 248, 235-240 (1992).

17. A. Arcangeli, A. Becchetti, A. Mannini, G. Mugnai, P. Defilippi, G. Tarone, M. R. Del Bene, E. Barletta, E. Wanke, M. Olivotto, Integrin-mediated neurite outgrowth in neuroblastoma cells depends on the activation of potassium channels. J. Cell Biol. 122, 1131-1143 (1993).

18. B. Lin, A. C. Arai, G. Lynch, C. M. Gall, Integrins regulate NMDA receptor-mediated synaptic currents. J. Neurophysiol. 89, 2874-2878 (2003).

19. J. A. Bernard-Trifilo, E. A. Kramár, R. Torp, C.-Y. Lin, E. A. Pineda, G. Lynch, C. M. Gall, Integrin signaling cascades are operational in adult hippocampal synapses and modulate NMDA receptor physiology. J. Neurochem. 93, 834-849 (2005).

20. Y. Yang, X. Wu, P. Gui, J. Wu, J. Z. Sheng, S. Ling, A. P. Braun, G. E. Davis, M. J. Davis, $\alpha .5 \beta 1$ integrin engagement increases large conductance, $\mathrm{Ca}^{2+}$-activated $\mathrm{K}^{+}$channel current and $\mathrm{Ca}^{2+}$ sensitivity through c-src-mediated channel phosphorylation. J. Biol. Chem. 285, 131-141 (2010).

21. M. Levite, L. Cahalon, A. Peretz, R. Hershkoviz, A. Sobko, A. Ariel, R. Desai, B. Attali, O. Lider, Extracellular $\mathrm{K}^{+}$and opening of voltage-gated potassium channels activate $\mathrm{T}$ cell integrin function: Physical and functional association between Kv1.3 channels and $\beta 1$ integrins. J. Exp. Med 191, 1167-1176 (2000).

22. D. J. Burkin, J. E. Kim, M. Gu, S. J. Kaufman, Laminin and $\alpha 7 \beta 1$ integrin regulate agrininduced clustering of acetylcholine receptors. J. Cell Sci. 113, 2877-2886 (2000).

23. A. Cherubini, G. Hofmann, S. Pillozzi, L. Guasti, O. Crociani, E. Cilia, M. Balzi, S. Degani, P. Di Stefano, P. Defilippi, E. Wanke, A. Becchetti, M. Olivotto, R. Wymore, A. Arcangeli, hERG1 channels are physically linked to $\beta 1$ integrins and modulate adhesion-dependent signaling. Mol. Biol. Cell 16, 2972-2983 (2005).

24. J.-T. Chao, P. Gui, G. W. Zamponi, G. E. Davis, M. J. Davis, Spatial association of the CaV1.2 calcium channel with $\alpha_{5} \beta_{1}$-integrin. Am. J. Physiol. Cell Physiol. 300, C477-C489 (2011).

25. K. Pozo, L. A. Cingolani, S. Bassani, F. Laurent, M. Passafaro, Y. Goda, $\beta 3$ integrin interacts directly with GluA2 AMPA receptor subunit and regulates AMPA receptor expression in hippocampal neurons. Proc. Natl. Acad. Sci. U.S.A. 109, 1323-1328 (2013).

26. O. Crociani, F. Zanieri, S. Pillozzi, E. Lastraioli, M. Stefanini, A. Fiore, A. Fortunato, M. D'Amico, M. Masselli, E. De Lorenzo, L. Gasparoli, M. Chiu, O. Bussolati, A. Becchetti, A. Arcangeli, hERG1 channels modulate integrin signaling to trigger angiogenesis and tumor progression in colorectal cancer. Sci. Rep. 3, 3308-3316 (2013).

27. S. Pillozzi, M. F. Brizzi, P. A. Bernabei, B. Bartolozzi, R. Caporale, V. Basile, V. Boddi, L. Pegoraro, A. Becchetti, A. Arcangeli, VEGFR-1 (FLT-1), $\beta_{1}$ integrin, and hERG K ${ }^{+}$channel form a macromolecular signaling complex in acute myeloid leukemia: Role in cell migration and clinical outcome. Blood 110, 1238-1250 (2007).

28. S. Pillozzi, M. Masselli, E. De Lorenzo, B. Accordi, E. Cilia, O. Crociani, A. Amedei, M. Veltroni, M. D'Amico, G. Basso, A. Becchetti, D. Campana, A. Arcangeli, Chemotherapy resistance in acute lymphoblastic leukemia requires hERG1 channels and is overcome by hERG1 blockers. Blood 117, 902-914 (2011).

29. F. S. Wouters, P. I. H. Bastiaens, R. W. A. Wirtz, T. M. Jovin, FRET microscopy demonstrates molecular association of non-specific lipid transfer protein (nsL-TP) WITH fatty acid oxidation enzymes in peroxisomes. EMBO J. 17, 7179-7189 (1998).

30. R. Hallworth, B. Currall, M. G. Nichols, X. Wu, J. Zuo, Studying inner ear protein-protein interaction using FRET and FLIM. Brain Res. 1091, 122-131 (2006).

31. S. Engel, S. Scolari, B. Thaa, N. Krebs, T. Korte, A. Herrmann, M. Veit, FLIM-FRET and FRAP reveal association of influenza virus haemagglutinin with membrane rafts. Biochem. J. 425, 567-573 (2006).

32. A. Pietraszewska-Bogiel, T. W. J. Gadella, FRET microscopy: From principle to routine technology in cell biology. J. Microsc. 241, 111-118 (2011).

33. X. Wang, R. Xu, G. Abernathey, J. Taylor, M. B. Alzghoul, K. Hannon, G. H. Hockerman, A. L. Pond, Kv11.1 channel subunit composition includes MinK and varies developmentally in mouse cardiac muscle. Dev. Dyn. 237, 2430-2437 (2008).

34. D. Nosi, R. Mercatelli, F. Chellini, S. Soria, A. Pini, L. Formigli, F. Quercioli, A molecular imaging analysis of $\mathrm{C} \times 43$ association with Cdo during skeletal myoblast differentiation. J. Biophotonics 6, 612-621 (2013).

35. S. Kupershmidt, D. J. Snyders, A. Raes, D. M. Roden, A K ${ }^{+}$channel splice variant common in human heart lacks a $\mathrm{C}$-terminal domain required for expression of rapidly activating delayed rectifier current. J. Biol. Chem. 273, 27231-27235 (1998). 
36. C. G. Viloria, F. Barros, T. Giráldez, D. Gómez-Varela, P. de la Peña, Differential effects of amino-terminal distal and proximal domains in the regulation of human erg $\mathrm{K}^{+}$channel gating. Biophys. J. 79, 231-246 (2000).

37. R. Fässler, M. Pfaff, J. Murphy, A. A. Noegel, S. Johansson, R. Timpl, R. Albrecht, Lack of beta 1 integrin gene in embryonic stem cells affects morphology, adhesion, and migration but not integration into the inner cell mass of blastocysts. J. Cell Biol. 128, 979-988 (1995).

38. Z. Es-Salah-Lamoureux, P. Y. Xiong, S. J. Goodchild, C. A. Ahern, D. Fedida, Blockade of permeation by potassium but normal gating of the G628S nonconducting hERG channel mutant. Biophys. J. 101, 662-670 (2011).

39. M. Zhang, J. Liu, G.-N. Tseng, Gating charges in the activation and inactivation processes of the hERG channel. J. Gen. Physiol. 124, 703-718 (2004).

40. I. M. Herzberg, M. C. Trudeau, G. A. Robertson, Transfer of rapid inactivation and sensitivity to the class III antiarrhythmic drug E-4031 from HERG to M-eag channels. J. Physiol. 511, 3-14 (1998).

41. L. Guasti, O. Crociani, E. Redaelli, S. Pillozzi, S. Polvani, M. Masselli, T. Mello, A. Galli, A. Amedei, R. S. Wymore, E. Wanke, A. Arcangeli, Identification of a posttranslational mechanism for the regulation of $\mathrm{hERG} 1 \mathrm{~K}^{+}$channel expression and hERG1 current density in tumor cells. Mol. Cell Biol. 28, 5043-5060 (2008).

42. F. J. Sulzmaier, C. Jean, D. D. Schlaepfer, FAK in cancer: Mechanistic findings and clinical applications. Nat. Rev. Cancer 14, 598-610 (2014).

43. G. H. Patterson, D. W. Piston, B. G. Barisas, Förster distances between green fluorescent protein pairs. Anal. Biochem. 284, 438-440 (2000).

44. J. Zhu, J. Zhu, T. A. Springer, Complete integrin headpiece opening in eight steps. J. Cell Biol. 201, 1053-1068 (2013).

45. C. Kim, F. Ye, X. Hu, M. H. Ginsberg, Talin activates integrins by altering the topology of the $\beta$ transmembrane domain. J. Cell Biol. 197, 605-611 (2012).

46. V. P. Hytönen, B. Wehrle-Haller, Mechanosensing in cell-matrix adhesions-Converting tensions into chemical signals. Exp. Cell Res. 343, 35-41 (2016).

47. F. Balzac, S. F. Retta, A. Albini, A. Melchiorri, V. E. Koteliansky, M. Geuna, L. Silengo, $\mathrm{G}$. Tarone, Expression of beta $1 \mathrm{~B}$ integrin isoform in $\mathrm{CHO}$ cells results in a dominant negative effect on cell adhesion and motility. J. Cell Biol. 127, 557-565 (1994).

48. L. Smilenov, R. Briesewitz, E. E. Marcantonio, Integrin $\beta 1$ cytoplasmic domain dominant negative effects revealed by lysophosphatidic acid treatment. Mol. Biol. Cell 5, 1215-1223 (1994).

49. T. Nagai, K. Ibata, E. S. Park, M. Kubota, K. Mikoshiba, A. Miyawaki, A variant of yellow fluorescent protein with fast and efficient maturation for cell-biological applications. Nat. Biotechnol. 20, 87-90 (2002)

50. S. Pillozzi, M. F. Brizzi, M. Balzi, O. Crociani, A. Cherubini, L. Guasti, B. Bartolozzi, A. Becchetti, E. Wanke, P. A. Bernabei, M. Olivotto, L. Pegoraro, A. Arcangeli, HERG potassium channels are constitutively expressed in primary human acute myeloid leukemias and regulate cell proliferation of normal and leukemic hemopoietic progenitors. Leukemia 16, 1791-1798 (2002).

51. F. Quercioli, B. Tiribilli, M. Vassalli, A. Ghirelli, Laser spectral characterization in multiphoton microscopy. Appl. Opt. 43, 3055-3060 (2004).

52. H. van Hoorn, R. Harkes, E. M. Spiesz, C. Storm, D. van Noort, B. Ladoux, T. Schmidt, The nanoscale architecture of force-bearing focal adhesions. Nano Lett. 14, 4257-4262 (2014).

53. L. Gasparoli, M. D’Amico, M. Masselli, S. Pillozzi, R. Caves, R. Khuwaileh, W. Tiedke, K. Mugridge, A. Pratesi, J. S. Mitcheson, G. Basso, A. Becchetti, A. Arcangeli, New pyrimidoindole compound CD-160130 preferentially inhibits the $\mathrm{K}_{\mathrm{V}} 11.1 \mathrm{~B}$ isoform and produces antileukemic effects without cardio-toxicity. Mol. Pharmacol. 87, 183-196 (2015).

54. V. S. LeBleu, J. T. O'Connell, K. N. Gonzalez Herrera, H. Wikman, K. Pantel, M. C. Haigis, F. Machado de Carvalho, A. Damascena, L. T. Domingos Chinen, R. M. Rocha, J. M. Asara, R. Kalluri, PGC- $1 \alpha$ mediates mitochondrial biogenesis and oxydative phosphorylation in cancer cells to promote metastasis. Nat. Cell Biol. 16, 992-1003 (2014).

55. A. L. Pond, B. K. Scheve, A. T. Benedict, K. Petrecca, D. R. Van Wagoner, A. Shrier, J. M. Nerbonne, Expression of distinct ERG proteins in rat, mouse and human heart. Relation to functional $I_{\mathrm{Kr}}$ channels. J. Biol. Chem. 275, 5997-6006 (2000).

56. E. Lastraioli, L. Guasti, O. Crociani, S. Polvani, G. Hofmann, H. Witchel, L. Bencini, M. Calistri, L. Messerini, M. Scatizzi, R. Moretti, E. Wanke, M. Olivotto, G. Mugnai, A. Arcangeli, herg1 gene and HERG1 protein are overexpressed in colorectal cancers and regulate cell invasion of tumor cells. Cancer Res. 64, 606-611 (2004).
57. R. A. Bhat, J. W. Borst, M. Riehl, R. D. Thompson, Interaction of maize Opaque-2 and the transcriptional co-activators GCN5 and ADA2, in the modulation of transcriptional activity. Plant Mol. Biol. 55, 239-252 (2004).

58. R. A. Bhat, M. Miklis, E. Schmelzer, P. Schulze-Lefert, R. Panstruga, Recruitment and interaction dynamics of plant penetration resistance components in a plasma membrane microdomain. Proc. Natl. Acad. Sci. U.S.A. 102, 3135-3140 (2005).

59. D. Llères, J. James, S. Swift, D. G. Norman, A. I. Lamond, Quantitative analysis of chromatin compaction in living cells using FLIM-FRET. J. Cell Biol. 187, 481-496 (2009).

60. A. Villoing, M. Ridhoir, B. Cinquin, M. Erard, L. Alvarez, G. Vallverdu, P. Pernot, R. Grailhe, F. Mérola, H. Pasquier, Complex fluorescence of the cyan fluorescent protein: Comparisons with the $\mathrm{H} 148 \mathrm{D}$ variant and consequences for quantitative cell imaging. Biochemistry 47, 12483-12492 (2008).

61. D. L. Spector, R. D. Goldman, Constructing and expressing fluorescent protein fusions. Cold Spring Harb. Protoc. 2010, pdb.top87 (2010).

62. G. Valentin, C. Verheggen, T. Piolot, H. Neel, M. Coppey-Moisan, E. Bertrand, Photoconversion of YFP into a CFP-like species during acceptor photobleaching FRET experiment. Nat. Methods 2, 801 (2005).

63. R. G. Immink, T. W. Gadella Jr., S. Ferrario, M. Busscher, G. C. Angenent, Analysis of MADS box protein-protein interactions in living plant cells. Proc. Natl. Acad. Sci. U.S.A. 99, 2416-2421 (2002).

64. G. W. Zack, W. E. Rogers, S. A. Latt, Automatic measurement of sister chromatid exchange frequency. J. Histochem. Cytochem. 25, 741-753 (1977).

65. C. Berney, G. Danuser, FRET or no FRET: A quantitative comparison. Biophys. J. 84, 3992-4010 (2003).

Acknowledgments: We thank J. M. Mitcheson (University of Leicester, U.K.) for providing the hERG1-G628S, hERG1-K525C, and hERG1-R531C plasmids; C. G. Viloria (University of Oviedo, Spain) for providing the $\Delta 2-370$ plasmid; T. Pozzan (University of Padua, Italy) for providing the pCS2Venus vector; S. LaFlamme (Albany Medical College, NY) for providing the $\beta_{1}$-cyto construct; J. Hancox (University of Bristol, U.K.) for providing the S620T plasmid; and L. Tofani for revising the statistical procedures. Funding: This work was supported by grants from Associazione Italiana per la Ricerca sul Cancro (\#10275 and \#15627 to A.A.), the Association for International Cancer Research (\#06-0491 to A.A.), lonTraC Grant FP7People-2011-ITN (\#289648 to A.A. and fellowship to S.M.), Associazione Genitori Noi per Voi (to A.A.), FRRB-Nanofarmaci and the University of Milano-Bicocca (to A.B.), the University of Firenze (to A.A.), and the Italian Foundation for Cancer Research Fellowship (\#16334 to S.C.), Author contributions: A.A. and A.B. designed and supervised the whole study; S. Crescioli, F.Z., S.P., O.C., G.P., A.F., and S.M. performed coimmunoprecipitation and Western blot experiments; R.M. and S. Coppola performed and analyzed FRET experiments; L.G., M.D., and A.B. performed patch-clamp data; L.C. and V.M. prepared hERG1 $\triangle \mathrm{C}+\mathrm{RD}$ and YFP constructs; S. Crescioli, F.Z., and A.F. prepared HEK-hERG1 mutants; A.A. prepared HCT116KCNE1, HEK-KCNE1, and MDA-MB-231 cells transfected with hERG1 mutants; O.C. prepared slides for FRET experiments; M.F.B., D.R., and M.R. provided human heart samples; F.Z. and A.M. produced HEK cells for HS-FLIM-FRET experiments; S.P., M.S., and A.A. performed in vivo experiments; G.P. and S.P. analyzed mouse tissue samples; T.S. and F.Q. revised FRET data; P.D. supervised coimmunoprecipitation data; A.A. and A.B. wrote the paper; and all authors commented on the manuscript. Competing interests: The authors declare that they have no competing interests.

Submitted 26 January 2016

Resubmitted 13 September 2016

Accepted 16 March 2017

Published 4 April 2017

10.1126/scisignal.aaf3236

Citation: A. Becchetti, S. Crescioli, F. Zanieri, G. Petroni, R. Mercatelli, S. Coppola, L. Gasparoli, M. D’Amico, S. Pillozzi, O. Crociani, M. Stefanini, A. Fiore, L. Carraresi, V. Morello, S. Manoli, M. F. Brizzi, D. Ricci, M. Rinaldi, A. Masi, T. Schmidt, F. Quercioli, P. Defilippi, A. Arcangeli, The conformational state of hERG1 channels determines integrin association, downstream signaling, and cancer progression. Sci. Signal. 10, eaaf3236 (2017). 
Science Signaling

MAAAS
The conformational state of hERG1 channels determines integrin association, downstream signaling, and cancer progression

Andrea Becchetti, Silvia Crescioli, Francesca Zanieri, Giulia Petroni, Raffaella Mercatelli, Stefano Coppola, Luca Gasparoli, Massimo D'Amico, Serena Pillozzi, Olivia Crociani, Matteo Stefanini, Antonella Fiore, Laura Carraresi, Virginia Morello, Sagar Manoli, Maria Felice Brizzi, Davide Ricci, Mauro Rinaldi, Alessio Masi, Thomas Schmidt, Franco Quercioli, Paola Defilippi and Annarosa Arcangeli (April 4, 2017)

Science Signaling 10 (473), . [doi: 10.1126/scisignal.aaf3236]

The following resources related to this article are available online at http://stke.sciencemag.org.

This information is current as of April 5, 2017.

Article Tools Visit the online version of this article to access the personalization and article tools:

http://stke.sciencemag.org/content/10/473/eaaf3236

\section{Supplemental Materials}

Related Content

References

Permissions
"Supplementary Materials"

http://stke.sciencemag.org/content/suppl/2017/03/31/10.473.eaaf3236.DC1

The editors suggest related resources on Science's sites: http://stke.sciencemag.org/content/sigtrans/7/312/ra15.full http://stke.sciencemag.org/content/sigtrans/7/309/ra7.full http://stke.sciencemag.org/content/sigtrans/2/77/ra32.full http://science.sciencemag.org/content/sci/323/5914/642.full

This article cites 64 articles, 32 of which you can access for free at: http://stke.sciencemag.org/content/10/473/eaaf3236\#BIBL

Obtain information about reproducing this article: http://www.sciencemag.org/about/permissions.dtl

Science Signaling (ISSN 1937-9145) is published weekly, except the last December, by the American Association for the Advancement of Science, 1200 New York Avenue, NW, Washington, DC 20005. Copyright 2017 by the American Association for the Advancement of Science; all rights reserved. 